\title{
A Study of Three-Prong Tau Decays with Charged Kaons
}

\section{The OPAL Collaboration}

\begin{abstract}
From an analysis of the ionisation energy loss of charged particles selected from a sample of $147926 \mathrm{e}^{+} \mathrm{e}^{-} \rightarrow \tau^{+} \tau^{-}$candidates recorded in the OPAL detector at $\mathrm{e}^{+} \mathrm{e}^{-}$centre-of-mass energies near the $\mathrm{Z}^{0}$ resonance, we determine the branching ratios:

$$
\begin{aligned}
\operatorname{Br}\left(\tau^{-} \rightarrow \nu_{\tau} \mathrm{K}^{-} \pi^{-} \pi^{+}\left(\pi^{0}\right)\right) & =0.343 \pm 0.073 \pm 0.031 \% \\
\operatorname{Br}\left(\tau^{-} \rightarrow \nu_{\tau} \mathrm{K}^{-} \pi^{-} \mathrm{K}^{+}\left(\pi^{0}\right)\right) & =0.159 \pm 0.053 \pm 0.020 \%
\end{aligned}
$$

where the $\left(\pi^{0}\right)$ notation refers to decay modes with or without an accompanying $\pi^{0}$. The $\tau^{-} \rightarrow \nu_{\tau} \mathrm{K}^{-} \pi^{-} \pi^{+}\left(\pi^{0}\right)$ final states occurring through $\tau^{-} \rightarrow \nu_{\tau} \mathrm{K}^{-} \mathrm{K}_{\mathrm{S}}^{0}\left(\pi^{0}\right)$ are treated as background in this analysis.

We also examine the resonant structure of $\tau^{-} \rightarrow \nu_{\tau} \mathrm{K}^{-} \pi^{-} \pi^{+}$candidates. Under the assumption that the resonant structure is dominated by the $\mathrm{K}_{1}$ resonances, we determine:

$$
R=\frac{\operatorname{Br}\left(\tau^{-} \rightarrow \nu_{\tau} \mathrm{K}_{1}(1270)\right)}{\operatorname{Br}\left(\tau^{-} \rightarrow \nu_{\tau} \mathrm{K}_{1}(1400)\right)+\operatorname{Br}\left(\tau^{-} \rightarrow \nu_{\tau} \mathrm{K}_{1}(1270)\right)}=0.71 \pm 0.16 \pm 0.11
$$
\end{abstract}

In all results, the first uncertainties are statistical and the second are systematic.

(Submitted to European Physical Journal C) 


\section{The OPAL Collaboration}

G. Abbiendi ${ }^{2}$, K. Ackerstaff ${ }^{8}$, G. Alexander ${ }^{23}$, J. Allison ${ }^{16}$, K.J. Anderson ${ }^{9}$, S. Anderson ${ }^{12}$, S. Arcelli ${ }^{17}$, S. Asai ${ }^{24}$, S.F. Ashby ${ }^{1}$, D. Axen ${ }^{29}$, G. Azuelos ${ }^{18, a}$, A.H. Ball ${ }^{8}$, E. Barberio ${ }^{8}$, R.J. Barlow ${ }^{16}$, J.R. Batley ${ }^{5}$, S. Baumann ${ }^{3}$, J. Bechtluft ${ }^{14}$,

T. Behnke ${ }^{27}$, K.W. Bell ${ }^{20}$, G. Bella ${ }^{23}$, A. Bellerive ${ }^{9}$, S. Bentvelsen ${ }^{8}$, S. Bethke ${ }^{14}$,

S. Betts ${ }^{15}$, O. Biebel ${ }^{14}$, A. Biguzzi ${ }^{5}$, I.J. Bloodworth ${ }^{1}$, P. Bock ${ }^{11}$, J. Böhme ${ }^{14}$, O. Boeriu ${ }^{10}$, D. Bonacorsi ${ }^{2}$, M. Boutemeur ${ }^{33}$, S. Braibant ${ }^{8}$, P. Bright-Thomas ${ }^{1}$, L. Brigliadori ${ }^{2}$, R.M. Brown ${ }^{20}$, H.J. Burckhart ${ }^{8}$, P. Capiluppi ${ }^{2}$, R.K. Carnegie ${ }^{6}$, A.A. Carter $^{13}$, J.R. Carter ${ }^{5}$, C.Y. Chang ${ }^{17}$, D.G. Charlton ${ }^{1, b}$, D. Chrisman ${ }^{4}$, C. Ciocca ${ }^{2}$, P.E.L. Clarke ${ }^{15}$, E. Clay $^{15}$, I. Cohen ${ }^{23}$, J.E. Conboy ${ }^{15}$, O.C. Cooke ${ }^{8}$, J. Couchman ${ }^{15}$, C. Couyoumtzelis ${ }^{13}$, R.L. Coxe ${ }^{9}$, M. Cuffiani ${ }^{2}$, S. Dado ${ }^{22}$, G.M. Dallavalle ${ }^{2}$, S. Dallison ${ }^{16}$, R. Davis ${ }^{30}$, S. De Jong ${ }^{12}$, A. de Roeck ${ }^{8}$, P. Dervan ${ }^{15}$, K. Desch ${ }^{27}$, B. Dienes ${ }^{32, h}$, M.S. Dixit ${ }^{7}$, M. Donkers ${ }^{6}$, J. Dubbert ${ }^{33}$, E. Duchovni ${ }^{26}$, G. Duckeck ${ }^{33}$, I.P. Duerdoth ${ }^{16}$, P.G. Estabrooks ${ }^{6}$, E. Etzion ${ }^{23}$, F. Fabbri ${ }^{2}$, A. Fanfani ${ }^{2}$, M. Fanti ${ }^{2}$, A.A. Faust ${ }^{30}$, L. Feld ${ }^{10}$, P. Ferrari ${ }^{12}$, F. Fiedler ${ }^{27}$, M. Fierro ${ }^{2}$, I. Fleck ${ }^{10}$, A. Frey ${ }^{8}$, A. Fürtjes ${ }^{8}$, D.I. Futyan ${ }^{16}$, P. Gagnon ${ }^{7}$, J.W. Gary ${ }^{4}$, G. Gaycken ${ }^{27}$, C. Geich-Gimbel ${ }^{3}$, G. Giacomelli ${ }^{2}$, P. Giacomelli ${ }^{2}$, W.R. Gibson ${ }^{13}$, D.M. Gingrich ${ }^{30, a}$, D. Glenzinski ${ }^{9}$, J. Goldberg ${ }^{22}$, W. Gorn ${ }^{4}$, C. Grandi ${ }^{2}$, K. Graham ${ }^{28}$, E. Gross ${ }^{26}$, J. Grunhaus ${ }^{23}$, M. Gruwé ${ }^{27}$, C. Hajdu ${ }^{31}$ G.G. Hanson ${ }^{12}$, M. Hansroul ${ }^{8}$, M. Hapke ${ }^{13}$, K. Harder ${ }^{27}$, A. Harel ${ }^{22}$, C.K. Hargrove ${ }^{7}$, M. Harin-Dirac ${ }^{4}$, M. Hauschild ${ }^{8}$, C.M. Hawkes ${ }^{1}$, R. Hawkings ${ }^{27}$, R.J.Hemingway ${ }^{6}$, G. Herten ${ }^{10}$, R.D.Heuer ${ }^{27}$, M.D. Hildreth ${ }^{8}$, J.C. Hill ${ }^{5}$, P.R. Hobson ${ }^{25}$, A.Hocker ${ }^{9}$, K. Hoffman ${ }^{8}$, R.J.Homer ${ }^{1}$, A.K. Honma ${ }^{28, a}$, D. Horváth ${ }^{31, c}$, K.R. Hossain ${ }^{30}$, R. Howard ${ }^{29}$, P. Hüntemeyer ${ }^{27}$, P. Igo-Kemenes ${ }^{11}$,

D.C. Imrie ${ }^{25}$, K. Ishii ${ }^{24}$, F.R. Jacob ${ }^{20}$, A. Jawahery ${ }^{17}$, H. Jeremie ${ }^{18}$, M. Jimack ${ }^{1}$, C.R. Jones ${ }^{5}$, P. Jovanovic ${ }^{1}$, T.R. Junk ${ }^{6}$, N. Kanaya ${ }^{24}$, J. Kanzaki ${ }^{24}$, D. Karlen ${ }^{6}$, V. Kartvelishvili ${ }^{16}$, K. Kawagoe ${ }^{24}$, T. Kawamoto ${ }^{24}$, P.I. Kayal ${ }^{30}$, R.K. Keeler ${ }^{28}$, R.G.Kellogg ${ }^{17}$, B.W.Kennedy ${ }^{20}$, D.H. Kim ${ }^{19}$, A. Klier ${ }^{26}$, T. Kobayashi' ${ }^{24}$, M. Kobel ${ }^{3, d}$, T.P. Kokott ${ }^{3}$, M. Kolrep ${ }^{10}$, S. Komamiya ${ }^{24}$, R.V.Kowalewski ${ }^{28}$, T. Kress ${ }^{4}$, P. Krieger ${ }^{6}$, J.von Krogh ${ }^{11}$, T. Kuhl ${ }^{3}$, P. Kyberd ${ }^{13}$, G.D. Lafferty ${ }^{16}$, H. Landsman ${ }^{22}$, D. Lanske ${ }^{14}$,

J. Lauber ${ }^{15}$, I. Lawson ${ }^{28}$, J.G. Layter ${ }^{4}$, D. Lellouch ${ }^{26}$, J. Letts ${ }^{12}$, L. Levinson ${ }^{26}$,

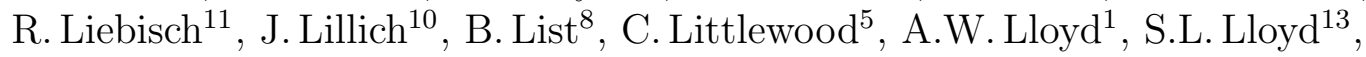
F.K. Loebinger ${ }^{16}$, G.D. Long $^{28}$, M.J. Losty ${ }^{7}$, J. Lu ${ }^{29}$, J. Ludwig ${ }^{10}$, D. Liu ${ }^{12}$, A. Macchiolo ${ }^{18}$, A. Macpherson ${ }^{30}$, W. Mader ${ }^{3}$, M. Mannelli $^{8}$, S. Marcellini' ${ }^{2}$, T.E. Marchant ${ }^{16}$, A.J. Martin ${ }^{13}$, J.P. Martin ${ }^{18}$, G. Martinez ${ }^{17}$, T. Mashimo ${ }^{24}$, P. Mättig ${ }^{26}$, W.J. McDonald ${ }^{30}$, J. McKenna ${ }^{29}$, E.A. Mckigney ${ }^{15}$, T.J. McMahon ${ }^{1}$, R.A. McPherson ${ }^{28}$, F. Meijers ${ }^{8}$, P. Mendez-Lorenzo ${ }^{33}$, F.S. Merritt ${ }^{9}$, H. Mes ${ }^{7}$, I. Meyer ${ }^{5}$, A. Michelini' ${ }^{2}$, S. Mihara ${ }^{24}$, G. Mikenberg ${ }^{26}$, D.J. Miller ${ }^{15}$, W. Mohr ${ }^{10}$, A. Montanari²,

T. Mori ${ }^{24}$, K. Nagai ${ }^{8}$, I. Nakamura ${ }^{24}$, H.A. Neal ${ }^{12, g}$, R. Nisius ${ }^{8}$, S.W. O’Neale ${ }^{1}$, F.G. Oakham ${ }^{7}$, F. Odorici ${ }^{2}$, H.O. Ogren ${ }^{12}$, A. Okpara ${ }^{11}$, M.J. Oreglia ${ }^{9}$, S. Orito ${ }^{24}$, G. Pásztor ${ }^{31}$, J.R. Pater ${ }^{16}$, G.N.Patrick ${ }^{20}$, J. Patt ${ }^{10}$, R. Perez-Ochoa ${ }^{8}$, S. Petzold ${ }^{27}$, P. Pfeifenschneider ${ }^{14}$, J.E. Pilcher ${ }^{9}$, J. Pinfold ${ }^{30}$, D.E. Plane ${ }^{8}$, P. Poffenberger ${ }^{28}$, 


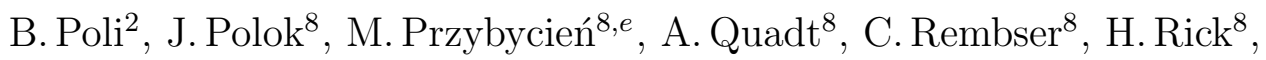
S. Robertson ${ }^{28}$, S.A. Robins ${ }^{22}$, N. Rodning ${ }^{30}$, J.M. Roney ${ }^{28}$, S. Rosati ${ }^{3}$, K. Roscoe ${ }^{16}$, A.M. Rossi ${ }^{2}$, Y.Rozen ${ }^{22}$, K. Runge ${ }^{10}$, O. Runolfsson ${ }^{8}$, D.R. Rust ${ }^{12}$, K. Sachs ${ }^{10}$, T.Saeki ${ }^{24}$, O.Sahr ${ }^{33}$, W.M.Sang ${ }^{25}$, E.K.G.Sarkisyan ${ }^{23}$, C.Sbarra ${ }^{29}$, A.D. Schaile ${ }^{33}$, O. Schaile ${ }^{33}$, P.Scharff-Hansen ${ }^{8}$, J.Schieck ${ }^{11}$, S. Schmitt ${ }^{11}$, A. Schöning ${ }^{8}$, M. Schröder ${ }^{8}$, M. Schumacher ${ }^{3}$, C. Schwick ${ }^{8}$, W.G. Scott ${ }^{20}$, R. Seuster ${ }^{14}$, T.G.Shears ${ }^{8}$, B.C. Shen ${ }^{4}$, C.H.Shepherd-Themistocleous ${ }^{5}$, P.Sherwood ${ }^{15}$, G.P. Siroli ${ }^{2}$, A. Skuja ${ }^{17}$, A.M. Smith ${ }^{8}$, G.A.Snow ${ }^{17}$, R. Sobie ${ }^{28}$, S. Söldner-Rembold ${ }^{10, f}$, S. Spagnolo ${ }^{20}$, M. Sproston ${ }^{20}$, A. Stahl ${ }^{3}$, K. Stephens ${ }^{16}$, K. Stoll ${ }^{10}$, D. Strom ${ }^{19}$, R. Ströhmer ${ }^{33}$, B. Surrow ${ }^{8}$, S.D. Talbot ${ }^{1}$, P. Taras ${ }^{18}$, S. Tarem ${ }^{22}$, R. Teuscher ${ }^{9}$, M. Thiergen ${ }^{10}$, J. Thomas ${ }^{15}$, M.A. Thomson ${ }^{8}$, E. Torrence ${ }^{8}$, S. Towers ${ }^{6}$, T. Trefzger ${ }^{33}$, I. Trigger ${ }^{18}$, Z. Trócsányi ${ }^{32, h}$, E. Tsur ${ }^{23}$, M.F. Turner-Watson ${ }^{1}$, I. Ueda ${ }^{24}$, R. Van Kooten ${ }^{12}$, P. Vannerem ${ }^{10}$, M. Verzocchi ${ }^{8}$, H. Voss $^{3}$, F. Wäckerle ${ }^{10}$, A. Wagner ${ }^{27}$, D. Waller 6 , C.P. Ward ${ }^{5}$, D.R. Ward ${ }^{5}$, P.M. Watkins ${ }^{1}$, A.T. Watson ${ }^{1}$, N.K. Watson ${ }^{1}$, P.S. Wells ${ }^{8}$, N. Wermes ${ }^{3}$, D. Wetterling ${ }^{11}$ J.S. White ${ }^{6}$, G.W.Wilson ${ }^{16}$, J.A. Wilson ${ }^{1}$, T.R. Wyatt ${ }^{16}$, S. Yamashita ${ }^{24}$, V.Zacek ${ }^{18}$, D. Zer-Zion ${ }^{8}$

${ }^{1}$ School of Physics and Astronomy, University of Birmingham, Birmingham B15 2TT, UK

${ }^{2}$ Dipartimento di Fisica dell' Università di Bologna and INFN, I-40126 Bologna, Italy ${ }^{3}$ Physikalisches Institut, Universität Bonn, D-53115 Bonn, Germany

${ }^{4}$ Department of Physics, University of California, Riverside CA 92521, USA

${ }^{5}$ Cavendish Laboratory, Cambridge CB3 0HE, UK

${ }^{6}$ Ottawa-Carleton Institute for Physics, Department of Physics, Carleton University, Ottawa, Ontario K1S 5B6, Canada

${ }^{7}$ Centre for Research in Particle Physics, Carleton University, Ottawa, Ontario K1S 5B6, Canada

${ }^{8}$ CERN, European Organisation for Particle Physics, CH-1211 Geneva 23, Switzerland ${ }^{9}$ Enrico Fermi Institute and Department of Physics, University of Chicago, Chicago IL 60637, USA

${ }^{10}$ Fakultät für Physik, Albert Ludwigs Universität, D-79104 Freiburg, Germany

${ }^{11}$ Physikalisches Institut, Universität Heidelberg, D-69120 Heidelberg, Germany

${ }^{12}$ Indiana University, Department of Physics, Swain Hall West 117, Bloomington IN 47405, USA

${ }^{13}$ Queen Mary and Westfield College, University of London, London E1 4NS, UK

${ }^{14}$ Technische Hochschule Aachen, III Physikalisches Institut, Sommerfeldstrasse 26-28, D-52056 Aachen, Germany

${ }^{15}$ University College London, London WC1E 6BT, UK

${ }^{16}$ Department of Physics, Schuster Laboratory, The University, Manchester M13 9PL, $\mathrm{UK}$

${ }^{17}$ Department of Physics, University of Maryland, College Park, MD 20742, USA

${ }^{18}$ Laboratoire de Physique Nucléaire, Université de Montréal, Montréal, Quebec H3C 
3J7, Canada

${ }^{19}$ University of Oregon, Department of Physics, Eugene OR 97403, USA

${ }^{20}$ CLRC Rutherford Appleton Laboratory, Chilton, Didcot, Oxfordshire OX11 0QX, $\mathrm{UK}$

${ }^{22}$ Department of Physics, Technion-Israel Institute of Technology, Haifa 32000, Israel

${ }^{23}$ Department of Physics and Astronomy, Tel Aviv University, Tel Aviv 69978, Israel

${ }^{24}$ International Centre for Elementary Particle Physics and Department of Physics, University of Tokyo, Tokyo 113-0033, and Kobe University, Kobe 657-8501, Japan

${ }^{25}$ Institute of Physical and Environmental Sciences, Brunel University, Uxbridge, Middlesex UB8 3PH, UK

${ }^{26}$ Particle Physics Department, Weizmann Institute of Science, Rehovot 76100, Israel

${ }^{27}$ Universität Hamburg/DESY, II Institut für Experimental Physik, Notkestrasse 85, D-22607 Hamburg, Germany

${ }^{28}$ University of Victoria, Department of Physics, P O Box 3055, Victoria BC V8W 3P6, Canada

${ }^{29}$ University of British Columbia, Department of Physics, Vancouver BC V6T 1Z1, Canada

${ }^{30}$ University of Alberta, Department of Physics, Edmonton AB T6G 2J1, Canada

${ }^{31}$ Research Institute for Particle and Nuclear Physics, H-1525 Budapest, P O Box 49, Hungary

${ }^{32}$ Institute of Nuclear Research, H-4001 Debrecen, P O Box 51, Hungary

${ }^{33}$ Ludwigs-Maximilians-Universität München, Sektion Physik, Am Coulombwall 1, D85748 Garching, Germany

${ }^{a}$ and at TRIUMF, Vancouver, Canada V6T 2A3

${ }^{b}$ and Royal Society University Research Fellow

${ }^{c}$ and Institute of Nuclear Research, Debrecen, Hungary

${ }^{d}$ on leave of absence from the University of Freiburg

$e$ and University of Mining and Metallurgy, Cracow

$f$ and Heisenberg Fellow

${ }^{g}$ now at Yale University, Dept of Physics, New Haven, USA

$h$ and Department of Experimental Physics, Lajos Kossuth University, Debrecen, Hungary.

\section{Introduction}

The study of rare decays of the tau lepton has been made possible by the availability of large, low background tau-pair samples, such as those produced at the $\mathrm{Z}^{0}$ resonance at LEP I. This paper reports on an analysis of three-prong tau decay modes contain- 
ing charged kaons, using the complete set of data collected by the OPAL experiment between 1990 and 1995 at $\mathrm{e}^{+} \mathrm{e}^{-}$centre-of-mass energies near the $\mathrm{Z}^{0}$ resonance. The excellent charged particle identification capability of the OPAL detector is exploited to study these states and obtain measurements of their branching ratios.

The $\tau^{ \pm}$lepton can only decay to hadronic final states of strangeness 0 or \pm 1 . These two allowed values of the final state strangeness imply that if a first-order weak decay of a tau to a three-prong final state includes exactly one kaon, then that kaon, if it is charged, must have the same charge as the initial tau. Likewise, if a first-order weak decay of a tau to a three-prong final state includes exactly two charged kaons, those kaons must be of opposite charge. Thus $\tau^{-} \rightarrow \nu_{\tau} \mathrm{K}^{-} \pi^{-} \pi^{+}$and $\tau^{-} \rightarrow \nu_{\tau} \mathrm{K}^{-} \pi^{-} \mathrm{K}^{+}$decays can both occur as first-order weak interactions. Decays which violate the strangeness constraint, such as $\tau^{-} \rightarrow \nu_{\tau} \pi^{-} \pi^{-} \mathrm{K}^{+}$and $\tau^{-} \rightarrow \nu_{\tau} \mathrm{K}^{-} \mathrm{K}^{-} \pi^{+}$, can only occur as second order weak interactions, and are therefore highly suppressed. The charge conjugate decays are implied in these interactions and throughout this paper.

Table 1 lists three-prong decays of the tau with charged kaons in the final state, along with the current experimental measurements and theoretical expectations for each decay branching ratio [1]-[7]. Due to the relative rarity of tau three-prong final states with charged kaons, and the experimental difficulties involved in charged particle identification, the branching ratios and resonant structure of these decays are not well known. We determine the branching ratios for $\tau^{-} \rightarrow \nu_{\tau} \mathrm{K}^{-} \pi^{-} \pi^{+}\left(\pi^{0}\right)$ and $\tau^{-} \rightarrow \nu_{\tau} \mathrm{K}^{-} \pi^{-} \mathrm{K}^{+}\left(\pi^{0}\right)$, and, to verify that the charged particle identification techniques used in this analysis yield unbiased branching ratio measurements, we also determine the branching ratio to the $\tau^{-} \rightarrow \nu_{\tau} \pi^{-} \pi^{-} \mathrm{K}^{+}\left(\pi^{0}\right)$ final state to ensure that the result is consistent with zero. In addition, we examine the resonant structure of these decays, and, under the assumption that the $\tau^{-} \rightarrow \nu_{\tau} \mathrm{K}^{-} \pi^{-} \pi^{+}$final state is dominated by the $\mathrm{K}_{1}$ intermediate resonances, we determine the branching ratio of $\tau^{-} \rightarrow \nu_{\tau} \mathrm{K}_{1}$ (1270) relative to the sum of the $\tau^{-} \rightarrow \nu_{\tau} \mathrm{K}_{1}(1270)$ and $\tau^{-} \rightarrow \nu_{\tau} \mathrm{K}_{1}(1400)$ branching ratios.

The $\tau^{-} \rightarrow \nu_{\tau} \mathrm{K}^{-} \pi^{-} \pi^{+}\left(\pi^{0}\right)$ final states occurring through $\tau^{-} \rightarrow \nu_{\tau} \mathrm{K}^{-} \mathrm{K}_{\mathrm{S}}^{0}\left(\pi^{0}\right)$ are considered background in the analysis presented here. In addition, three-prong tau decays with charged kaons and more than one $\pi^{0}$ are severely phase-space suppressed and are therefore neglected.

\section{The OPAL Detector}

The OPAL detector is described in detail in reference [8]. The component of the detector most important to this analysis is the central jet chamber, which measures the momentum and specific ionisation energy loss, $\mathrm{d} E / \mathrm{d} x$, of charged particles. The jet chamber provides the only means of distinguishing between charged pions and kaons in the momentum range of interest to this study. 
The jet chamber is a cylinder $4 \mathrm{~m}$ long and $3.7 \mathrm{~m}$ in diameter, and is divided by cathode wire planes into 24 azimuthal sectors. Each sector contains one radial plane of anode wires, which are staggered to resolve left-right ambiguities. The chamber is contained in a solenoidal magnetic field of $0.435 \mathrm{~T}$, and is filled with an argon-methaneisobutane gas mixture at a pressure of 4 atmospheres. This arrangement provides a transverse spatial resolution of $\sigma_{x y}=130 \mu \mathrm{m}$, and a two-hit resolution of $2.5 \mathrm{~mm}$. In the barrel region of the jet chamber $(|\cos \theta|<0.72)$ t, the ionisation energy loss of a charged particle is sampled up to 159 times. A truncated mean is formed by discarding the highest $30 \%$ of the measurements, resulting in a $\mathrm{d} E / \mathrm{d} x$ resolution of about $3 \%$ for isolated tracks in the chamber [9].

A layer of wire chambers with drift direction parallel to the $z$-axis lies immediately outside the jet chamber. These $z$-chambers accurately determine the polar angle of charged particles traversing the central detector. A lead-glass electromagnetic calorimeter and presampler chambers are located outside the magnetic coil and jet chamber pressure vessel. The electromagnetic calorimeter is primarily used in this analysis to identify tau decays which include a $\pi^{0}$ in the final state. The return yoke of the OPAL magnet is instrumented for hadron calorimetery, and is surrounded by external muon chambers. The hadron calorimeter and muon chambers are mainly used in this analysis to select control samples containing muons and to veto non-tau backgrounds in the tau-pair candidate sample.

\subsection{Parameterisation of Ionisation Energy Loss}

Figure 1(a) shows the dependence of $\mathrm{d} E / \mathrm{d} x$ on the momentum of various particles in the OPAL jet chamber, as predicted by the OPAL energy loss parameterisation, $D_{\text {pred }}$ [9]. Figure 1 (b) shows the particle resolving power, $\mathcal{R}_{i j}$, versus momentum for various pairs of particle species $i$ and $j$, where

$$
\mathcal{R}_{i j} \equiv\left|D_{\text {pred }}^{i}-D_{\text {pred }}^{j}\right| / \sigma,
$$

and where $\sigma$ is the mean of the uncertainties of $D_{\text {pred }}^{i}$ and $D_{\text {pred }}^{j}$. Note that the OPAL jet chamber yields a pion/kaon separation of at least $2 \sigma$ for particles between about 2 to $30 \mathrm{GeV} / \mathrm{c}$.

The parameters used in the calculation of $D_{\text {pred }}$ and $\sigma$ are tuned to the measured energy loss of charged particles in hadronic $\mathrm{Z}^{0}$ decays, yielding a $\mathrm{d} E / \mathrm{d} x$ parameterisation that is accurate enough for nearly all analyses of OPAL data.

\footnotetext{
${ }^{1} \mathrm{~A}$ spherical coordinate system is used, with the $+z$-axis in the direction of the circulating electron beam. The angle $\theta$ is defined as the polar angle with respect to the $+z$-axis, and $\phi$ is defined as the azimuthal angle measured from the $+x$-axis, which points towards the centre of the LEP ring.
} 
However, hadronic tau decays are characterised by lower multiplicity and narrower jets than those produced in hadronic $\mathrm{Z}^{0}$ decays, resulting in small systematic differences between the measured $\mathrm{d} E / \mathrm{d} x$ in the two environments. Although these differences are less than one $\sigma$ in magnitude, they must be corrected for accurate particle identification in three-prong tau decays. Based on a study of the measured energy loss of muons in $\mathrm{e}^{+} \mathrm{e}^{-} \rightarrow \mu^{+} \mu^{-}, \tau^{-} \rightarrow \mu^{-} \bar{\nu}_{\mu} \nu_{\tau}$, and $\mathrm{e}^{+} \mathrm{e}^{-} \rightarrow \mathrm{e}^{+} \mathrm{e}^{-} \mu^{+} \mu^{-}$samples, the following corrections are applied to the measured energy loss, $D_{\text {meas }}$, and to the OPAL $\mathrm{d} E / \mathrm{d} x$ parameterisation and its uncertainty, $D_{\text {pred }}$ and $\sigma$ :

Multiplicative correction to $D_{\text {pred }}$ : To obtain a parameterisation which correctly predicts the measured energy loss of particles from tau decays, $D_{\text {pred }}$ must be corrected with a multiplicative factor, $s(\beta)$. Parameterising $s(\beta)$ as a second order polynomial in $Q(\beta)=-\log \left(1-\beta^{2}\right)$ yields satisfactory results. The magnitude of the correction is of order $1 \%$ for tracks in one-prong tau decays.

Additive correction to $D_{\text {meas }}$ : The measured energy loss of charged particles in lowmultiplicity events is found to depend strongly on the azimuthal separation, $\phi$, between the track and anode plane in the jet chamber cell. To improve the $\mathrm{d} E / \mathrm{d} x$ resolution, this behaviour is corrected with a function, $f(\phi)$, with seven parameters tuned to the measured energy loss of $\mathrm{e}^{+} \mathrm{e}^{-} \rightarrow \mu^{+} \mu^{-}$candidates. The magnitude of this correction is also of order $1 \%$.

Multiplicative correction to $\sigma$ : To correctly predict the spread in the $\mathrm{d} E / \mathrm{d} x$ of charged particles from one-prong tau decays, the OPAL parameterisation of $\sigma$ must be corrected with a multiplicative factor, $s_{\text {res }} \approx 0.9$.

Although the application of these corrections significantly improves the parameterisation of $\mathrm{d} E / \mathrm{d} x$ in a low multiplicity environment, the energy loss distribution contains a significant non-Gaussian component. This feature is most apparent in the corrected $\mathrm{d} E / \mathrm{d} x$ stretch distribution of the unlike-sign tracks of tau decays classified as $\tau^{-} \rightarrow \nu_{\tau} \pi^{-} \pi^{-} \mathrm{X}^{+}\left(\pi^{0}\right)$ 2. Stretch energy loss, $S_{i}$, is defined as:

$$
S_{i}=\left(D_{\text {meas }}-D_{\text {pred }}^{i}\right) / \sigma^{i}
$$

where $i$ is the particle hypothesis used to calculate the predicted $\mathrm{d} E / \mathrm{d} x$ and its uncertainty. The $\tau^{-} \rightarrow \nu_{\tau} \pi^{-} \pi^{-} \mathrm{X}^{+}\left(\pi^{0}\right)$ control sample is selected using $S_{\mathrm{K}}>2.0$ to identify three-prong tau events with two like-sign pion candidates in the final state. Since the $\tau^{-} \rightarrow \nu_{\tau} \pi^{-} \pi^{-} \mathrm{K}^{+}\left(\pi^{0}\right)$ final states are highly suppressed, the unlike-sign tracks in this sample are expected to be over $99.7 \%$ pure in pions. The stretch $\mathrm{d} E / \mathrm{d} x$ distribution under a pion hypothesis of this sample is seen in Figure 2 (a).

\footnotetext{
${ }^{2}$ The unlike-sign track is the track in the three-prong decay with charge opposite that of the initial tau.
} 
The non-Gaussian component is large in the region of the $\mathrm{d} E / \mathrm{d} x$ distribution expected to be populated by kaons. Parameterisation of the non-Gaussian component would therefore lead to very large statistical and systematic uncertainties in the measurement of the $\tau^{-} \rightarrow \nu_{\tau} \mathrm{K}^{-} \pi^{-} \pi^{+}\left(\pi^{0}\right)$ and $\tau^{-} \rightarrow \nu_{\tau} \mathrm{K}^{-} \pi^{-} \mathrm{K}^{+}\left(\pi^{0}\right)$ branching ratios. The tail is dramatically reduced in the $\mathrm{d} E / \mathrm{d} x$ distribution of tracks in three-prong tau decays which lie closest to the anode plane, as shown in Figure 2(b). When tracks are spatially close together in the jet chamber cell, the tail of the pulse from the track closest to the anode will effectively shift the pedestal of the pulse of the second track, leading to an incorrect determination of deposited charge for that track, and the observed tails in the $\mathrm{d} E / \mathrm{d} x$ distribution. The low-end non-Gaussian tail exists in the measured energy loss of charged particles in hadronic $\mathrm{Z}^{0}$ events, but is more pronounced in three-prong tau decays due to the tighter collimation of the particles in the decay.

The simplest method to minimize this effect is to only consider the $\mathrm{d} E / \mathrm{d} x$ of the track in the three-prong tau decay closest to the anode plane. Tracks meeting this requirement will be referred to as the 'first-track' three-prong sample. Further, the effect of pulses following the first is reduced by only considering first pulses separated from the second by more than $1 \mathrm{~cm}[9]$.

The corrected stretch $\mathrm{d} E / \mathrm{d} x$ distribution of unlike-sign tracks in the first-track $\tau^{-} \rightarrow \nu_{\tau} \pi^{-} \pi^{-} \mathrm{X}^{+}$sample, seen in Figure 2(b), is consistent with a Gaussian of zero mean and unit width.

\section{Monte Carlo Generated Event Samples}

For this analysis, tau lepton decays are simulated with the KORALZ 4.0 Monte Carlo generator and the Tauola 2.4 decay package [10] [11. To estimate biases introduced to the branching ratios by the tau-pair pre-selection procedure, we use a Monte Carlo sample of 600000 tau-pair events that have input branching ratios determined from world averages or theoretical expectations. In order to determine the branching ratios and to study the intermediate resonant structure of $\tau^{-} \rightarrow \nu_{\tau} \mathrm{K}^{-} \pi^{-} \pi^{+}$ and $\tau^{-} \rightarrow \nu_{\tau} \mathrm{K}^{-} \pi^{-} \mathrm{K}^{+}$decays, large Monte Carlo samples of signal events are also produced. All Monte Carlo samples are passed through a detailed simulation of the OPAL detector [12] and are subjected to the same analysis chain as the data.

The $\tau^{-} \rightarrow \nu_{\tau} \mathrm{K}^{-} \pi^{-} \pi^{+}$decay mode is generated in Tauola 2.4 primarily through the $\mathrm{K}_{1}(1400)$ intermediate resonance:

$$
\begin{aligned}
& \tau^{-} \rightarrow \nu_{\tau} \mathrm{K}_{1}(1400) \rightarrow \nu_{\tau} \mathrm{K}^{*}(892) \pi^{-} \rightarrow \nu_{\tau} \mathrm{K}^{-} \pi^{-} \pi^{+} \\
& \tau^{-} \rightarrow \nu_{\tau} \mathrm{K}_{1}(1400) \rightarrow \nu_{\tau} \rho(770) \mathrm{K}^{-} \rightarrow \nu_{\tau} \mathrm{K}^{-} \pi^{-} \pi^{+}
\end{aligned}
$$


However, the resonant structure of $\tau^{-} \rightarrow \nu_{\tau} \mathrm{K}^{-} \pi^{-} \pi^{+}$decays is likely more diverse than that represented in (1) and (2), as the decay can also occur through the $\mathrm{K}_{1}(1270)$ intermediate resonance [13]. The $\mathrm{K}_{1}(1270)$ predominantly decays to $\mathrm{K}^{*}(892) \pi, \mathrm{K}_{0}^{*}(1430) \pi$, and $\rho(770) \mathrm{K}$ [14]. To assess the sensitivity of the efficiency estimation to the assumptions made about the $\tau^{-} \rightarrow \nu_{\tau} \mathrm{K}^{-} \pi^{-} \pi^{+}$intermediate resonant structure, a modified version of Tauola is used to generate the following decays:

$$
\begin{aligned}
& \tau^{-} \rightarrow \nu_{\tau} \mathrm{K}_{1}(1270) \rightarrow \nu_{\tau} \mathrm{K}^{*}(892) \pi^{-} \rightarrow \nu_{\tau} \mathrm{K}^{-} \pi^{-} \pi^{+} \\
& \tau^{-} \rightarrow \nu_{\tau} \mathrm{K}_{1}(1270) \rightarrow \nu_{\tau} \mathrm{K}_{0}^{*}(1430) \pi^{-} \rightarrow \nu_{\tau} \mathrm{K}^{-} \pi^{-} \pi^{+} \\
& \tau^{-} \rightarrow \nu_{\tau} \mathrm{K}_{1}(1270) \rightarrow \nu_{\tau} \rho(770) \mathrm{K}^{-} \rightarrow \nu_{\tau} \mathrm{K}^{-} \pi^{-} \pi^{+}
\end{aligned}
$$

In each case, the $\mathrm{K}_{1}(1270)$ decays through a Breit-Wigner resonance with an sdependent width, and phase space limitations are taken into account [15]. Other $\mathrm{K}_{1}(1270)$ decay modes are ignored and interference effects between the various decay chains are neglected. Based upon experimental measurements of the $\mathrm{K}_{1}(1270)$ partial widths [14, the $\mathrm{K}_{1}(1270)$ is assumed to decay $49 \%$ of the time to $K \rho, 32 \%$ of the time to $K_{0}^{*}(1430) \pi$, and $19 \%$ of the time to $\mathrm{K}^{*}(892) \pi$.

A sample of $\tau^{-} \rightarrow \nu_{\tau} \mathrm{K}^{-} \pi^{-} \pi^{+}$events is also generated through the $\mathrm{K}_{1}(1400)$ resonance, again with the $\mathrm{K}_{1}$ decaying through a Breit-Wigner resonance with an $s$ dependent width. We only allow the $\mathrm{K}_{1}(1400)$ 's in this sample to decay to $\mathrm{K}^{*}(892) \pi$, as experimental measurements indicate that this decay mode occurs much more frequently than the $\rho(770) \mathrm{K}$ mode [16].

The $\tau^{-} \rightarrow \nu_{\tau} \mathrm{K}^{-} \pi^{-} \mathrm{K}^{+}$decay mode is generated in Tauola 2.4 through a mixture of the $\mathrm{a}_{1}(1260)$ and $\rho(1700)$ intermediate resonances:

$$
\begin{aligned}
& \tau^{-} \rightarrow \nu_{\tau} \mathrm{a}_{1}(1260) \rightarrow \nu_{\tau} \mathrm{K}^{*}(892) \mathrm{K}^{-} \rightarrow \nu_{\tau} \mathrm{K}^{-} \pi^{-} \mathrm{K}^{+} \\
& \tau^{-} \rightarrow \nu_{\tau} \rho(1700) \rightarrow \nu_{\tau} \mathrm{K}^{*}(892) \mathrm{K}^{-} \rightarrow \nu_{\tau} \mathrm{K}^{-} \pi^{-} \mathrm{K}^{+}
\end{aligned}
$$

Since the intermediate structure of the $\nu_{\tau} \mathrm{K}^{-} \pi^{-} \pi^{+} \pi^{0}$ and $\nu_{\tau} \mathrm{K}^{-} \pi^{-} \mathrm{K}^{+} \pi^{0}$ tau decay final states is not well understood theoretically, and experimental data on these decays are sparse, the Monte Carlo samples for these channels are generated through non-resonant production only. As a cross-check to ensure that the results of the $\tau^{-} \rightarrow \nu_{\tau} \mathrm{K}^{-} \pi^{-} \pi^{+}$and $\tau^{-} \rightarrow \nu_{\tau} \mathrm{K}^{-} \pi^{-} \mathrm{K}^{+}$resonant studies are independent of the modelling of the $\tau^{-} \rightarrow \nu_{\tau} \mathrm{K}^{-} \pi^{-} \pi^{+} \pi^{0}$ and $\tau^{-} \rightarrow \nu_{\tau} \mathrm{K}^{-} \pi^{-} \mathrm{K}^{+} \pi^{0}$ backgrounds, the following Monte Carlo samples are also used:

$$
\begin{aligned}
& \tau^{-} \rightarrow \nu_{\tau} \mathrm{K}^{-} \omega \rightarrow \nu_{\tau} \mathrm{K}^{-} \pi^{-} \pi^{+} \pi^{0} \\
& \tau^{-} \rightarrow \nu_{\tau}\left(\mathrm{K}^{*}(892) \mathrm{K} \pi\right)^{-} \rightarrow \nu_{\tau} \mathrm{K}^{-} \pi^{-} \mathrm{K}^{+} \pi^{0} .
\end{aligned}
$$

The second-order weak $\tau^{-} \rightarrow \nu_{\tau} \pi^{-} \pi^{-} \mathrm{K}^{+}\left(\pi^{0}\right)$ decays are modelled using Monte Carlo samples of $\tau^{-} \rightarrow \nu_{\tau} \mathrm{K}^{-} \pi^{-} \pi^{+}\left(\pi^{0}\right)$ decays, where the charge of the like-sign kaon and the unlike-sign pion are reversed. 
The efficiency corrections in the branching ratio analysis are estimated using $\tau^{-} \rightarrow \nu_{\tau} \mathrm{K}^{-} \pi^{-} \pi^{+}$and $\tau^{-} \rightarrow \nu_{\tau} \mathrm{K}^{-} \pi^{-} \mathrm{K}^{+}$events generated with the default version of Tauola 2.4, and $\tau^{-} \rightarrow \nu_{\tau} \mathrm{K}^{-} \pi^{-} \pi^{+} \pi^{0}$ and $\tau^{-} \rightarrow \nu_{\tau} \mathrm{K}^{-} \pi^{-} \mathrm{K}^{+} \pi^{0}$ events generated through non-resonant production. As a cross-check, the analysis is also performed with efficiency corrections derived from the $\tau^{-} \rightarrow \nu_{\tau} \mathrm{K}_{1}(1270)$ and $\tau^{-} \rightarrow \nu_{\tau} \mathrm{K}_{1}(1400)$ Monte Carlo samples.

\section{Branching Ratio Measurements}

\subsection{Event Selection}

This analysis uses full data set collected by the OPAL detector between the years 1990 and 1995 at $\mathrm{e}^{+} \mathrm{e}^{-}$centre-of-mass energies close to the $\mathrm{Z}^{0}$ resonance. Only data for which the tracking chambers and the electromagnetic calorimeter were fully operational are retained. The topology of $\mathrm{e}^{+} \mathrm{e}^{-} \rightarrow \tau^{+} \tau^{-}$events is characterised by a pair of backto-back, narrow jets with low particle multiplicity. Jets are defined in this analysis by grouping tracks and electromagnetic clusters into cones with an opening half-angle of $35^{\circ}$, where each cone is assumed to contain the decay products of one of the tau leptons. Tau-pair candidates are selected by requiring two low-multiplicity jets with an average polar angle of $\left|\cos \theta_{\text {ave }}\right|<0.90$. Background events from other lepton pairs, hadronic $\mathrm{Z}^{0}$ decays, and two-photon events are reduced with cuts on the event topology and total visible energy. These selections produce a sample of 147926 tau-pair candidates, with background $f^{\text {non }-\tau}=2.73 \pm 0.27 \%$, estimated by data control samples and Monte Carlo background samples. The tau-pair selection procedure is the same as that which is described in detail in reference [17], except for the average polar angle selection, which is extended in this analysis to include tracks in the endcap region of the OPAL detector.

The sum of the charges of the particles in each pre-selected tau-pair candidate event is required to be equal to zero. Three-prong tau decay candidates are selected from the jets in this sample containing three well-reconstructed charged tracks. The sum of the charges of the three tracks is required to be \pm 1 , and decays with $\mathrm{K}_{\mathrm{S}}^{0}$ candidates are excluded by rejecting tau decay candidates with one well-reconstructed neutral vertex with $\pi^{+} \pi^{-}$invariant mass between 0.4 and $0.6 \mathrm{GeV}$ [18]. The charge of the initial tau is taken to be the sum of the charges of the tracks in the cone associated with the tau lepton decay. This selection yields 38995 three-prong tau decay candidates. 


\subsubsection{Inclusive Candidate Samples (no $\pi^{0}$ Identification)}

The 'first-track' sample is formed by selecting the track in each three-prong tau decay candidate that is closest to the anode plane. This track is also required to lie within $|\cos \theta|<0.90$, and have at least 40 jet chamber hits used in the measurement of the $\mathrm{d} E / \mathrm{d} x$. All tracks in the first-track sample are also required to have reconstructed momentum between 3 and $90 \mathrm{GeV} / \mathrm{c}$. Tracks in the barrel region of the OPAL detector $(|\cos \theta|<0.72)$ are required to have at least 3 hits in the $z$-chambers. Tracks which cross another track within the volume of the OPAL jet chamber are rejected from this sample. The 'like-sign' and 'unlike-sign' track samples are formed by selecting tracks in the first-track sample that have the same and opposite charge as that of the initial tau, respectively. The number of tracks in these two samples are 9296 and 4722, respectively.

In first-order weak decays of the tau only the $\tau^{-} \rightarrow \nu_{\tau} \mathrm{K}^{-} \pi^{-} \mathrm{K}^{+}\left(\pi^{0}\right)$ decay modes contribute kaons to the unlike-sign sample, whereas both the $\tau^{-} \rightarrow \nu_{\tau} \mathrm{K}^{-} \pi^{-} \mathrm{K}^{+}\left(\pi^{0}\right)$ and $\tau^{-} \rightarrow \nu_{\tau} \mathrm{K}^{-} \pi^{-} \pi^{+}\left(\pi^{0}\right)$ decay modes contribute kaons to the like-sign sample. Thus, after corrections for efficiencies and subtraction of background kaons, the numbers of kaons in these two samples are used to deduce the branching ratios to the $\tau^{-} \rightarrow \nu_{\tau} \mathrm{K}^{-} \pi^{-} \pi^{+}\left(\pi^{0}\right)$ and $\tau^{-} \rightarrow \nu_{\tau} \mathrm{K}^{-} \pi^{-} \mathrm{K}^{+}\left(\pi^{0}\right)$ final states.

As a consistency check, we verify that the $\tau^{-} \rightarrow \nu_{\tau} \pi^{-} \pi^{-} \mathrm{K}^{+}\left(\pi^{0}\right)$ final states are indeed suppressed. To do this, we form a sample depleted in $\tau^{-} \rightarrow \nu_{\tau} \mathrm{K}^{-} \pi^{-} \mathrm{K}^{+}\left(\pi^{0}\right)$ decays from the unlike-sign sample by requiring both associated like-sign tracks to have $S_{\pi}>-1.0$. The $K^{-} \pi^{-} K^{+}$-enhanced sample is formed from tracks in the unlikesign sample which do no pass this selection. The number of tracks in these two samples is 1527 and 3195, respectively. The numbers of kaons in the $K^{-} \pi^{-} K^{+}$-depleted sample, the $K^{-} \pi^{-} K^{+}$-enhanced sample, and the like-sign sample are used to simultaneously determine the tau branching fractions to the $\nu_{\tau} \mathrm{K}^{-} \pi^{-} \pi^{+}\left(\pi^{0}\right), \nu_{\tau} \mathrm{K}^{-} \pi^{-} \mathrm{K}^{+}\left(\pi^{0}\right)$, and $\nu_{\tau} \pi^{-} \pi^{-} \mathrm{K}^{+}\left(\pi^{0}\right)$ final states.

\subsection{2 $\pi^{0}$ Identification}

To determine the branching ratios for decays with and without $\pi^{0}$ 's, we divide all the inclusive samples into separate $\pi^{0}$-enhanced and $\pi^{0}$-depleted samples using $\sum E / \sum P>$ 0.5 and $\sum E / \sum P \leq 0.5$, respectively, where $\sum E / \sum P$ is the sum of the energy deposited in the electromagnetic calorimeter, divided by the scalar sum of the momentum of the charged particles in the tau decay cone. The $\sum E / \sum P$ distributions for the Monte Carlo $\tau^{-} \rightarrow \nu_{\tau} \mathrm{K}^{-} \pi^{-} \pi^{+}\left(\pi^{0}\right)$ and $\tau^{-} \rightarrow \nu_{\tau} \mathrm{K}^{-} \pi^{-} \mathrm{K}^{+}\left(\pi^{0}\right)$ samples are shown in Figure 3 . The number of tracks in the $\pi^{0}$-depleted samples with the like-sign, unlike-sign, and $K^{-} \pi^{-} K^{+}$-depleted selections are 5984, and 3188 , and 1110 , respectively. 


\subsection{Estimation of the Number of Kaons in the Samples}

To determine the number of kaons in the data candidate samples described in Sections 4.1.1 and 4.1.2, we maximise a likelihood function based on $\mathrm{d} E / \mathrm{d} x$ :

$$
\mathcal{L}=\prod_{j=1, N} \sum_{i=\mathrm{e}, \pi, \mathrm{K}} f_{i} W^{i j}
$$

where $W^{i j}$ is the $\mathrm{d} E / \mathrm{d} x$ weight of charged particle $j$ under particle hypothesis $i$,

$$
W^{i j}=\frac{1}{\sqrt{2 \pi} s^{\prime}\left(\beta_{j}\right) s_{\mathrm{res}} \sigma_{i j}} \exp \left[-\frac{1}{2}\left(\frac{D_{\text {meas }}^{j}-f\left(\phi_{j}\right)-s^{\prime}\left(\beta_{j}\right) D_{\mathrm{pred}}^{i j}}{s^{\prime}\left(\beta_{j}\right) s_{\mathrm{res}} \sigma_{i j}}\right)^{2}\right],
$$

and where

$f_{i}$ is the fraction of particle type $i$ in the sample, where $i$ is either pion, kaon, or electron. The values of $f_{i}$ are constrained to be non-negative, and the sum is constrained to be 1 .

$N$ is the total number of particles in the sample, $N=\left(N_{\mathrm{e}}+N_{\pi}+N_{\mathrm{K}}\right)$.

$D_{\text {meas }}^{j}$ is the measured $\mathrm{d} E / \mathrm{d} x$ of the $j^{\text {th }}$ charged particle.

$D_{\text {pred }}^{i j}$ is the predicted $\mathrm{d} E / \mathrm{d} x$ for charged particle $j$, calculated with the OPAL parameterisation under particle hypothesis $i$, as derived from the measured $\mathrm{d} E / \mathrm{d} x$ of charged particles in hadronic $\mathrm{Z}^{0}$ events.

$\sigma_{i j}$ is the uncertainty on $D_{\text {pred }}^{i j}$, calculated using the OPAL parameterisation, as derived from hadronic $\mathrm{Z}^{0}$ events.

$s_{\text {res }}$ is the multiplicative correction to $\sigma_{i j}$ for all particle hypotheses $i$, and is allowed to vary without constraint in each fit. Separate $s_{\text {res }}$ factors are used for charged particles in the barrel and endcap regions of the OPAL detector. The central values of these parameters are about 0.84 and 0.73 , respectively.

$s^{\prime}(\beta)$ is the $\beta$ dependent multiplicative correction to $D_{\text {pred }}$, and is equal to $s(\beta)+\alpha$, where $s(\beta)$ is determined from the one-prong control samples, and $\alpha$ is a correction allowed to vary in each fit to compensate for possible slight differences between the $\mathrm{d} E / \mathrm{d} x$ of the one-prong and three-prong decay environments. Separate $s^{\prime}(\beta)$ factors are needed for charged particles from three-prong tau decays in the barrel and endcap regions of the OPAL detector. These extra constant terms yield a correction to the $\mathrm{d} E / \mathrm{d} x$ of approximately $1 \%$.

$f(\phi)$ is the $\phi$ dependent correction to the measured $\mathrm{d} E / \mathrm{d} x$, as obtained from the one-prong control samples. 
Efforts are made to obtain a $\mathrm{d} E / \mathrm{d} x$ parameterisation for the tau decay environment that is optimal for many particle species over a wide range of momenta. However, it is possible that the $\mathrm{d} E / \mathrm{d} x$ corrections described in Section 2.1 may be somewhat more (or less) optimal for pions than they are for kaons in the momentum range of interest. Thus, to correct for any possible species-dependent quality differences in the parameterisation of $\mathrm{d} E / \mathrm{d} x$, an extra factor, $C_{\mathrm{K}}$, is allowed to multiply the kaon predicted energy loss, $D_{\mathrm{pred}}^{\mathrm{K}}$, in the likelihood function found in Equation 3. This factor is determined in a likelihood fit to the measured $\mathrm{d} E / \mathrm{d} x$ of one-prong tau decays to hadron final states to be $C_{\mathrm{K}}=0.9968 \pm 0.0014$.

Independent likelihood fits are performed for different ranges of momentum (13 bins of variable size between 3 and $90 \mathrm{GeV} / \mathrm{c}$ ). A test of the fit with large Monte Carlo samples verifies that the resulting estimates for the kaon fraction have biases within the range $-0.5 \sigma_{f_{\mathrm{K}}}$ to $0.3 \sigma_{f_{\mathrm{K}}}$ at the $95 \%$ confidence level, where $\sigma_{f_{\mathrm{K}}}$ is the typical statistical uncertainty on the kaon fraction returned by the fit. Thus, biases resulting from the fit procedure are neglected.

The number of kaons found within each of the samples is given in Table 2, and the stretch $\mathrm{d} E / \mathrm{d} x$ distributions of tracks in all momentum bins of the inclusive data like-sign and unlike-sign samples are shown in Figure $₫$. The normalisation of the predicted distributions of the kaons, pions, and electrons in each sample is obtained from the results of the likelihood fits. The $\chi^{2}$ per degree of freedom between the first 11 bins of the data and predicted distributions, which is the portion of the $\mathrm{d} E / \mathrm{d} x$ distribution populated by kaons, is 15.9/10 and 10.6/7 for the like-sign and unlike-sign samples, respectively.

\section{3 $\mathrm{d} E / \mathrm{d} x$ Systematic Studies}

A significant source of systematic uncertainty in this measurement is the uncertainty in the parameterisation of the predicted energy loss. To assess this systematic, we determine the sensitivity of the likelihood estimates of the number of kaons within each of the data samples to the uncertainties in the $\mathrm{d} E / \mathrm{d} x$ correction factors obtained from the one-prong samples. To achieve this, we modify the likelihood function from equation 3 such that:

$$
\mathcal{L}^{\prime}=\exp \left(-\frac{1}{2} \mathbf{n}^{\mathbf{T}} \mathbf{V}^{-1} \mathbf{n}\right) \mathcal{L}
$$

where

$\mathbf{n}$ is a vector containing the difference from the central values of the seven $\mathrm{d} E / \mathrm{d} x$ correction factors that describe the function $f(\phi)$, and the three correction factors that describe the function $s(\beta)$, where the central values are as derived from the fits to the one-prong control samples. 
$\mathbf{V}$ is the covariance matrix for the $\mathrm{d} E / \mathrm{d} x$ correction factors.

In the first iteration, the correction factors are allowed to vary in the fit, and the returned values are found to be consistent with the input values. In the second iteration, the likelihood fit is repeated, this time keeping the $\mathrm{d} E / \mathrm{d} x$ correction factors fixed to the values from the first iteration. The systematic uncertainty in $f_{\mathrm{K}}$ due to the parameterisation of $\mathrm{d} E / \mathrm{d} x$ is then obtained from the square root of the quadrature difference of the fit uncertainties in $f_{\mathrm{K}}$ from the two iterations, and is shown in Table 2 .

\subsection{Efficiency Correction}

The efficiencies for kaons from signal events in the pre-selected tau-pair sample to enter the candidate samples are estimated using signal events generated with the KORALZ 4.0 Monte Carlo generator and the Tauola 2.4 decay package, as described in Section 3.

Table 2 shows the average efficiency estimates for the various signal channels in the pre-selected tau decay sample that contribute kaons to the data samples described in Sections 4.1.1 and 4.1.2. All efficiencies are corrected for biases introduced by the tau-pair pre-selection using a Monte Carlo tau-pair sample, and the efficiency uncertainties include the systematic uncertainty arising from this correction. The preselection biases are $0.935 \pm 0.012,0.925 \pm 0.013,0.908 \pm 0.012$, and $0.898 \pm 0.013$ for $\tau^{-} \rightarrow \nu_{\tau} \mathrm{K}^{-} \pi^{-} \pi^{+}, \tau^{-} \rightarrow \nu_{\tau} \mathrm{K}^{-} \pi^{-} \mathrm{K}^{+}, \tau^{-} \rightarrow \nu_{\tau} \mathrm{K}^{-} \pi^{-} \pi^{+} \pi^{0}$, and $\tau^{-} \rightarrow \nu_{\tau} \mathrm{K}^{-} \pi^{-} \mathrm{K}^{+} \pi^{0}$ decays, respectively.

Figure 5 shows the efficiency versus momentum for $\tau^{-} \rightarrow \nu_{\tau} \mathrm{K}^{-} \pi^{-} \pi^{+}$and $\tau^{-} \rightarrow \nu_{\tau} \mathrm{K}^{-} \pi^{-} \mathrm{K}^{+}$in the pre-selected tau-pair sample to contribute kaons to the inclusive like-sign and unlike-sign samples, respectively. The branching ratio analysis is performed using an efficiency correction that is binned in momentum.

The variation of the branching ratios due to alternative intermediate resonant structure scenarios for the $\tau^{-} \rightarrow \nu_{\tau} \mathrm{K}^{-} \pi^{-} \pi^{+}$final state is assessed using efficiency estimates from signal events generated by the modified version of Tauola 2.4 through the $\mathrm{K}_{1}(1270)$ and $\mathrm{K}_{1}(1400)$ intermediate states as described in Section 3 . The mixture of $K_{1}(1270)$ and $K_{1}(1400)$ is taken from the results of the analysis of the intermediate resonant structure of $\tau^{-} \rightarrow \nu_{\tau} \mathrm{K}^{-} \pi^{-} \pi^{+}$candidates, as described in Section 5 , $R=\operatorname{Br}\left(\tau^{-} \rightarrow \nu_{\tau} \mathrm{K}_{1}(1270)\right) / \operatorname{Br}\left(\tau^{-} \rightarrow \nu_{\tau}\left(\mathrm{K}_{1}(1400)\right.\right.$ or $\left.\left.\mathrm{K}_{1}(1270)\right)\right)=0.71 \pm 0.19$. The branching ratios obtained within this range are found to be in agreement with the central values to within $1.5 \sigma$ of the combined Monte Carlo statistical uncertainty.

As a further cross-check, the branching ratios are evaluated using $\tau^{-} \rightarrow \nu_{\tau} \mathrm{K}^{-} \pi^{-} \pi^{+}$ and $\tau^{-} \rightarrow \nu_{\tau} \mathrm{K}^{-} \pi^{-} \mathrm{K}^{+}$efficiencies estimated using Monte Carlo events generated through 
non-resonant production only. The resulting branching ratios are in agreement with the branching ratio central values to within $1 \sigma$ of the combined Monte Carlo statistical uncertainty.

\subsection{Kaon Background Correction}

Background kaons in the data samples described in Sections 4.1.1 and 4.1.2 are estimated by applying the same selection criteria to Monte Carlo samples of hadronic $\mathrm{Z}^{0}$ decays and tau-pair decays. From the number of selected events that contain kaons, the estimated number of background kaons are derived, as summarised in Table 2 . Dominant sources of background kaons include low-multiplicity hadronic $\mathrm{Z}^{0}$ events, and $\tau^{-} \rightarrow \nu_{\tau} \mathrm{K}^{-} \geq 0$ neutrals decays.

\subsection{The Branching Ratio Calculation}

The branching ratios in the exclusive tau decay channels of interest are calculated from the numbers of kaons, as estimated by the likelihood fit, in several momentum bins of the exclusive candidate samples, listed in Table 2. The number of kaons within each momentum bin $i$ of candidate sample $j$ is corrected for background as described in Section 4.5, yielding

$$
R_{i j}=\frac{\left(N_{\mathrm{K}}^{i j}-N_{\mathrm{bkgnd}}^{i j}\right)}{N_{\tau}^{\mathrm{pre}-\mathrm{sel}}\left(1-f^{\text {non }-\tau}\right)},
$$

where

$N_{\tau}^{\text {pre-sel }}$ is the number of pre-selected tau decay candidates. There were 295852 tau decay candidates recorded in the OPAL detector between the years 1990 and 1995.

$f^{\text {non- }-\tau}$ is the estimated background from non- $\tau$ sources in the pre-selected tau decay candidates $\left(f^{\text {non }-\tau}=2.73 \pm 0.27 \%\right)$.

$N_{\mathrm{K}}^{i j}$ is the number of kaons in momentum bin $i$ of candidate sample $j$, as estimated by the likelihood fit. The number of kaons summed over all momentum bins of each sample is shown in Table 2 .

$N_{\text {bkgnd }}^{i j}$ is the estimated number of background kaons in momentum bin $i$ of candidate sample $j$, as estimated from Monte Carlo tau decay and hadronic $\mathrm{Z}^{0}$ decay samples. The number of background kaons summed over all momentum bins of each sample is shown in Table 2 . 
A set of linear equations that relate the $R_{i j}$ to the tau branching ratios are solved simultaneously to determine the branching ratios. The set of equations includes efficiency corrections as a function of momentum for each decay channel, as determined from Monte Carlo generated events, as described in Section 4.4. The efficiency correction does not assume that the momentum spectrum of the signal kaons in each sample follows those of kaons from the Monte Carlo. For background corrections, however, the momentum spectra of Monte Carlo generated events are used.

In the first step of the branching ratio calculation, the $\tau^{-} \rightarrow \nu_{\tau} \pi^{-} \pi^{-} \mathrm{K}^{+}\left(\pi^{0}\right)$ branching ratios are not assumed to be zero. The branching ratio calculation uses the division of the unlike-sign samples into the $\mathrm{K}^{-} \pi^{-} \mathrm{K}^{+}$-enhanced and $\mathrm{K}^{-} \pi^{-} \mathrm{K}^{+}$-depleted samples in conjunction with the like-sign samples, for a total of six exclusive samples, as listed in Table 2. The results of the calculation are:

$$
\begin{aligned}
& \operatorname{Br}\left(\tau^{-} \rightarrow \nu_{\tau} \mathrm{K}^{-} \pi^{-} \pi^{+}\left(\pi^{0}\right)\right)=+0.404 \pm 0.083 \% \\
& \operatorname{Br}\left(\tau^{-} \rightarrow \nu_{\tau} \mathrm{K}^{-} \pi^{-} \mathrm{K}^{+}\left(\pi^{0}\right)\right)=+0.066 \pm 0.079 \% \\
& \operatorname{Br}\left(\tau^{-} \rightarrow \nu_{\tau} \pi^{-} \pi^{-} \mathrm{K}^{+}\left(\pi^{0}\right)\right)=+0.077 \pm 0.070 \% \\
& \operatorname{Br}\left(\tau^{-} \rightarrow \nu_{\tau} \mathrm{K}^{-} \pi^{-} \pi^{+} \pi^{0}\right)=+0.064 \pm 0.086 \% \\
& \operatorname{Br}\left(\tau^{-} \rightarrow \nu_{\tau} \mathrm{K}^{-} \pi^{-} \mathrm{K}^{+} \pi^{0}\right)=-0.110 \pm 0.128 \% \\
& \operatorname{Br}\left(\tau^{-} \rightarrow \nu_{\tau} \pi^{-} \pi^{-} \mathrm{K}^{+} \pi^{0}\right)=+0.137 \pm 0.109 \% \\
& \operatorname{Br}\left(\tau^{-} \rightarrow \nu_{\tau} \mathrm{K}^{-} \pi^{-} \pi^{+}\right)=+0.340 \pm 0.086 \% \\
& \operatorname{Br}\left(\tau^{-} \rightarrow \nu_{\tau} \mathrm{K}^{-} \pi^{-} \mathrm{K}^{+}\right)=+0.176 \pm 0.078 \% \\
& \operatorname{Br}\left(\tau^{-} \rightarrow \nu_{\tau} \pi^{-} \pi^{-} \mathrm{K}^{+}\right)=-0.060 \pm 0.068 \% \text {, }
\end{aligned}
$$

where the uncertainties are statistical only, and where the inclusive branching ratios are obtained from the sums of the exclusive branching ratios. Correlations between the exclusive branching ratios are taken into account in this calculation. The results for the branching ratios to the $\nu_{\tau} \pi^{-} \pi^{-} \mathrm{K}^{+}\left(\pi^{0}\right)$ final states, which can only occur as second-order weak interactions, are consistent with zero, as expected, indicating that there are no significant biases in the kaon identification procedure.

To obtain more precise branching ratios for the first-order weak decays, we set the $\tau^{-} \rightarrow \nu_{\tau} \pi^{-} \pi^{-} \mathrm{K}^{+}\left(\pi^{0}\right)$ branching ratios to zero and repeat the calculation. In this iteration, the calculation uses the unlike-sign and like-sign exclusive samples, for a total of four samples. The results of this calculation are:

$$
\begin{aligned}
\operatorname{Br}\left(\tau^{-} \rightarrow \nu_{\tau} \mathrm{K}^{-} \pi^{-} \pi^{+}\left(\pi^{0}\right)\right) & =+0.343 \pm 0.073 \pm 0.031 \% \\
\operatorname{Br}\left(\tau^{-} \rightarrow \nu_{\tau} \mathrm{K}^{-} \pi^{-} \mathrm{K}^{+}\left(\pi^{0}\right)\right) & =+0.159 \pm 0.053 \pm 0.020 \% \\
\operatorname{Br}\left(\tau^{-} \rightarrow \nu_{\tau} \mathrm{K}^{-} \pi^{-} \pi^{+} \pi^{0}\right) & =-0.017 \pm 0.076 \pm 0.060 \%
\end{aligned}
$$




$$
\begin{aligned}
& \operatorname{Br}\left(\tau^{-} \rightarrow \nu_{\tau} \mathrm{K}^{-} \pi^{-} \mathrm{K}^{+} \pi^{0}\right)=+0.072 \pm 0.085 \pm 0.051 \% \\
& \operatorname{Br}\left(\tau^{-} \rightarrow \nu_{\tau} \mathrm{K}^{-} \pi^{-} \pi^{+}\right)=+0.360 \pm 0.082 \pm 0.048 \% \\
& \operatorname{Br}\left(\tau^{-} \rightarrow \nu_{\tau} \mathrm{K}^{-} \pi^{-} \mathrm{K}^{+}\right)=+0.087 \pm 0.056 \pm 0.040 \% \text {, }
\end{aligned}
$$

where the first uncertainties are statistical and the second are systematic. The summary of the systematic uncertainties for each branching ratio is shown in Table 3. The correlation matrix for the exclusive branching ratios is found in Table 4 .

The $\tau^{-} \rightarrow \nu_{\tau} \mathrm{K}^{-} \pi^{-} \pi^{+}\left(\pi^{0}\right)$ branching ratios from the first and second calculations are approximately $70 \%$ correlated, and the $\tau^{-} \rightarrow \nu_{\tau} \mathrm{K}^{-} \pi^{-} \mathrm{K}^{+}\left(\pi^{0}\right)$ branching ratios from the first and second calculations are approximately $50 \%$ correlated. In both cases, the resulting differences in the branching ratios between the two iterations are consistent to within $1.3 \sigma$ of the combined statistical uncertainties.

The central values of the branching ratios are evaluated using an $\sum E / \sum P$ selection of 0.5 to distinguish between states including and not including a $\pi^{0}$. The branching ratios are also evaluated using $\sum E / \sum P$ selections of $0.3,0.4,0.6$, and 0.7 . The branching ratio systematic uncertainty associated with the $\sum E / \sum P$ selection is taken as the RMS spread of the five values, and is quoted in Table 3 .

\section{Resonant Structure}

\subsection{Event Selection}

The selection of events used in the study of the resonant structure begins with the three-prong tau decay candidate sample described in Section 4. Events which include an $\mathrm{e}^{+} \mathrm{e}^{-}$pair from a photon converting in the detector material are identified and rejected on the basis of a topological conversion finder [18. All three tracks in each decay are required to have at least 40 jet chamber hits used in the measurement of $\mathrm{d} E / \mathrm{d} x$. Tracks in the barrel region of the OPAL detector are required to have at least 3 hits in the $z$-chambers.

Using $S_{\pi}<-2.0$ to identify kaons and $\left|S_{\pi}\right|<1.5$ to identify pions, events are classified into the following channels:

- $\tau^{-} \rightarrow \nu_{\tau} \mathrm{K}^{-} \pi^{-} \pi^{+}$candidates have one like-sign kaon, and an unlike-sign and likesign pion.

- $\tau^{-} \rightarrow \nu_{\tau} \mathrm{K}^{-} \pi^{-} \mathrm{K}^{+}$candidates have one like-sign pion, and an unlike-sign and likesign kaon. 
- $\tau^{-} \rightarrow \nu_{\tau} \pi^{-} \pi^{-} \pi^{+}\left(\pi^{0}\right)$ candidates have all three tracks identified as pions.

The number of kaons in the lowest momentum bin of the candidate samples used in the branching ratio analysis is found to be consistent with zero. Thus, to increase the signal to noise ratio in the samples used in the resonant studies, all kaon candidates are required to have momentum of at least $5 \mathrm{GeV} / \mathrm{c}$. Pion candidates are required to have momentum of at least $0.75 \mathrm{GeV} / \mathrm{c}$. The above selections produce $950 \tau^{-} \rightarrow \nu_{\tau} \mathrm{K}^{-} \pi^{-} \pi^{+}$ and $79 \tau^{-} \rightarrow \nu_{\tau} \mathrm{K}^{-} \pi^{-} \mathrm{K}^{+}$candidates.

The non-Gaussian tails in the data $\mathrm{d} E / \mathrm{d} x$ distribution are not as problematic in this portion of the analysis as they are in the branching ratio analysis and thus no attempt is made to remove them. The differing shapes of the signal and the $\tau^{-} \rightarrow \nu_{\tau} \pi^{-} \pi^{-} \pi^{+}\left(\pi^{0}\right)$ invariant mass distributions yield some separation power between signal and background in the data samples, and the $\mathrm{d} E / \mathrm{d} x$ selections used to identify pions and kaons in this study are designed only to provide three-prong tau decay samples enhanced with $\tau^{-} \rightarrow \nu_{\tau} \mathrm{K}^{-} \pi^{-} \pi^{+}$or $\tau^{-} \rightarrow \nu_{\tau} \mathrm{K}^{-} \pi^{-} \mathrm{K}^{+}$decays. Other than these initial selections, $\mathrm{d} E / \mathrm{d} x$ is not used to distinguish between signal and background.

\section{$5.2 \quad \tau^{-} \rightarrow \nu_{\tau} \mathrm{K}^{-} \pi^{-} \pi^{+}$}

The background in the $\tau^{-} \rightarrow \nu_{\tau} \mathrm{K}^{-} \pi^{-} \pi^{+}$sample consists primarily of $\tau^{-} \rightarrow \nu_{\tau} \pi^{-} \pi^{-} \pi^{+}\left(\pi^{0}\right)$ events, along with contributions from $\tau^{-} \rightarrow \nu_{\tau} \mathrm{K}^{-} \pi^{-} \pi^{+} \pi^{0}$, other tau decays, and hadronic $\mathrm{Z}^{0}$ events.

To analyse the resonant structure of these decays, we examine the $M_{\mathrm{K}^{-} \pi^{-} \pi^{+}}$, $M_{\mathrm{K}^{-} \pi^{+}}$, and $M_{\pi^{-} \pi^{+}}$invariant mass distributions of the candidates. To estimate the shape of the dominant three-pion background in these distributions, we use the data $\tau^{-} \rightarrow \nu_{\tau} \pi^{-} \pi^{-} \pi^{+}\left(\pi^{0}\right)$ candidate sample, with the $5 \mathrm{GeV} / \mathrm{c}$ momentum selection placed on the particle which corresponds to the like-sign kaon candidate. This selection produces a sample of about 16000 events. The shape of the $\tau^{-} \rightarrow \nu_{\tau} \mathrm{K}^{-} \pi^{-} \pi^{+} \pi^{0}$ background in the distributions is parameterised with Monte Carlo $\tau^{-} \rightarrow \nu_{\tau} \mathrm{K}^{-} \pi^{-} \pi^{+} \pi^{0}$ events generated through non-resonant production. Other background fractions are expected to be on the order of a percent or less and are neglected.

The description of the shape of the signal portion of these invariant mass distributions depends upon assumptions made about the intermediate resonant structure of $\tau^{-} \rightarrow \nu_{\tau} \mathrm{K}^{-} \pi^{-} \pi^{+}$decays. We consider four different scenarios:

1. The $\tau^{-} \rightarrow \nu_{\tau} \mathrm{K}^{-} \pi^{-} \pi^{+}$decays occur through a mixture of $\mathrm{K}_{1}(1270)$ and $\mathrm{K}_{1}(1400)$. For this study we assume that the $\mathrm{K}_{1}(1270)$ has a width of $90 \mathrm{MeV}$, and the $\mathrm{K}_{1}(1400)$ has a width of $174 \mathrm{MeV}$, the current world average widths of these resonances [14]. 
2. The $\tau^{-} \rightarrow \nu_{\tau} \mathrm{K}^{-} \pi^{-} \pi^{+}$decays occur through the $\mathrm{K}_{1}(1270)$ and $\mathrm{K}_{1}(1400)$ resonances, and that the widths of these resonances are both $300 \mathrm{MeV}$, as suggested in reference [5].

3. The $\tau^{-} \rightarrow \nu_{\tau} \mathrm{K}^{-} \pi^{-} \pi^{+}$decays occur through the $\mathrm{K}^{*}(892) \pi^{-}$and $\rho(770) K^{-}$intermediate resonances.

4. The $\tau^{-} \rightarrow \nu_{\tau} \mathrm{K}^{-} \pi^{-} \pi^{+}$decays occur through non-resonant production (phase space) only.

Monte Carlo samples having at least 10 times the statistics of the expected signal component of the data $\tau^{-} \rightarrow \nu_{\tau} \mathrm{K}^{-} \pi^{-} \pi^{+}$candidates are generated with a modified version of Tauola 2.4 under each of these resonant structure assumptions.

To estimate the background and signal fractions in a way which accounts for correlations between the invariant mass distributions, we divide the $M_{\mathrm{K}^{-} \pi^{-} \pi^{+}}, M_{\mathrm{K}^{-} \pi^{+}}$, and $M_{\pi^{-} \pi^{+}}$invariant masses of the data, signal Monte Carlo sets, and background samples into 7 bins each, to form a $7 \times 7 \times 7$ matrix. Further discrimination between signal and background is obtained by dividing the scalar sum of the momenta in each threeprong decay into 24 bins between 0 and $48 \mathrm{GeV} / \mathrm{c}$. The correlations between $\sum P$ and the invariant masses are not significant compared to the inter-correlations between the invariant masses.

Using the various signal and background distributions as templates, a binned maximum likelihood fit is performed simultaneously to the data $7 \times 7 \times 7$ matrix and the $\sum P$ distribution to determine the most probable fractions of signal and $\tau^{-} \rightarrow \nu_{\tau} \mathrm{K}^{-} \pi^{-} \pi^{+} \pi^{0}$ background in the $\tau^{-} \rightarrow \nu_{\tau} \mathrm{K}^{-} \pi^{-} \pi^{+}$candidates. For the first three scenarios, two signal fractions are allowed to float. Bins in the matrix and $\sum P$ distribution which contain data events but no predicted signal or background events are neglected in the fit 5 . A test of the procedure using a Monte Carlo event sample of the same size and approximate composition of the data sample reveals no significant bias in the estimates of the signal and background fractions from the fit.

The results of the fits are shown in Table 5. Table 5 also gives the $\chi^{2}$ per degree of freedom between the data and predicted $\sum P, M_{\mathrm{K}^{-} \pi^{-} \pi^{+}}, M_{\mathrm{K}^{-} \pi^{+}}$, and $M_{\pi^{-} \pi^{+}}$distributions, where the normalisation of the predicted distributions is obtained from the fit results. The binning of these distributions is the same as that seen in Figure 6, which displays the results of the fit to the data using the assumption of the world average widths for the two $K_{1}$ resonances. The correlation between the estimated fractions of $\mathrm{K}_{1}(1270)$ and $\mathrm{K}_{1}(1400)$ obtained from this fit is about -0.30 . A fit where the signal and $\tau^{-} \rightarrow \nu_{\tau} \mathrm{K}^{-} \pi^{-} \pi^{+} \pi^{0}$ fractions are set to zero yields $-2 \log \mathcal{L} / \mathcal{L}_{\max }=107$. Since this

\footnotetext{
${ }^{3}$ Only one such bin exists in the fit for the central values of the signal and background fractions, and it contains only one event.
} 
fit has three fewer degrees of freedom than the first, the difference in $-2 \log \mathcal{L}$ should be $\chi^{2}$ distributed with three degrees of freedom if the sample does indeed consist of only three-pion background. This test disfavours this hypothesis at a confidence level of over $99 \%$.

Table 5 includes estimates of the efficiencies for $\tau^{-} \rightarrow \nu_{\tau} \mathrm{K}^{-} \pi^{-} \pi^{+}$and $\tau^{-} \rightarrow \nu_{\tau} \mathrm{K}^{-} \pi^{-} \pi^{+} \pi^{0}$ in the pre-selected tau-pair sample to contribute to the $\tau^{-} \rightarrow \nu_{\tau} \mathrm{K}^{-} \pi^{-} \pi^{+}$candidate samples. These efficiencies are not corrected for biases introduced by the $\tau^{-} \rightarrow \nu_{\tau} \mathrm{K}^{-} \pi^{-} \pi^{+}$selection procedure. In order to verify that the signal fractions returned by the fit are reasonable, we use these efficiencies and the fit fractions to calculate estimates of the $\tau^{-} \rightarrow \nu_{\tau} \mathrm{K}^{-} \pi^{-} \pi^{+}$and $\tau^{-} \rightarrow \nu_{\tau} \mathrm{K}^{-} \pi^{-} \pi^{+} \pi^{0}$ branching ratios, which are also given in Table 5. Although the efficiencies have not been corrected for bias, these estimates are in agreement with the branching ratios obtained in Section 4 for all resonant structure assumptions, except for $\tau^{-} \rightarrow \nu_{\tau} \mathrm{K}^{-} \pi^{-} \pi^{+}$through phase space only.

As a cross-check, Monte Carlo generated $\tau^{-} \rightarrow \nu_{\tau} \mathrm{K}^{-} \omega$ decays are used to estimate the shape of the $\tau^{-} \rightarrow \nu_{\tau} \mathrm{K}^{-} \pi^{-} \pi^{+} \pi^{0}$ in the data distributions, rather than $\tau^{-} \rightarrow \nu_{\tau} \mathrm{K}^{-} \pi^{-} \pi^{+} \pi^{0}$ generated through non-resonant production. For all four assumptions made about the $\tau^{-} \rightarrow \nu_{\tau} \mathrm{K}^{-} \pi^{-} \pi^{+}$intermediate resonant structure, these fits return signal fractions within $0.15 \sigma$ of the central values, and $\tau^{-} \rightarrow \nu_{\tau} \mathrm{K}^{-} \pi^{-} \pi^{+} \pi^{0}$ fractions within $0.35 \sigma$ of the central values, where $\sigma$ refers to the statistical uncertainties of the central values of the fractions.

\subsection{1 $\tau^{-} \rightarrow \nu_{\tau} \mathrm{K}_{1}$}

Under the assumption that resonant structure of $\tau^{-} \rightarrow \nu_{\tau} \mathrm{K}^{-} \pi^{-} \pi^{+}$decays is dominated by the $\mathrm{K}_{1}$ intermediate resonances, we derive

$$
R_{\mathrm{fit}}=\frac{\text { fraction of } \mathrm{K}_{1}(1270) \text { in } \mathrm{K}^{-} \pi^{-} \pi^{+} \text {candidates }}{\text { fraction of } \mathrm{K}_{1}(1400)+\mathrm{K}_{1}(1270) \text { in } \mathrm{K}^{-} \pi^{-} \pi^{+} \text {candidates }}=0.69 \pm 0.16,
$$

where the uncertainty is statistical, and where the fractions of $K_{1}(1270)$ and $K_{1}(1400)$ are taken from the fit which assumes the widths of these resonances are $90 \mathrm{MeV}$ and $174 \mathrm{MeV}$, respectively.

The signal and background template distributions used in the binned likelihood fit have statistical uncertainties associated with them. To determine the systematic uncertainties on the fit fractions arising from these uncertainties, the fit is repeated 25 times, each time randomly varying each bin of the template distributions by sampling Poisson distributions with means equal to the original bin contents. The resulting systematic uncertainties are included in Table 5, and are derived from the RMS spread of the signal fraction estimates from the 25 fits. 
To check for undue variation that may be produced by the choice of binning used in the fit, the above procedure is repeated for various binning schemes. In all cases, the RMS variation of the results returned by the different fits is less than the statistical uncertainty from the original fit. The variation of $R_{\text {fit }}$ due to uncertainty in the $\mathrm{K}_{1}(1270)$ branching fractions is also studied, and found to be negligible.

The value of $R_{\text {fit }}$ is corrected for the efficiencies for $\mathrm{K}_{1}(1270)$ and $\mathrm{K}_{1}(1400)$ in the pre-selected tau-pair sample to contribute to the $\tau^{-} \rightarrow \nu_{\tau} \mathrm{K}^{-} \pi^{-} \pi^{+}$candidate sample $(0.052 \pm 0.001$ and $0.058 \pm 0.002$, respectively). This yields:

$$
R=\frac{\operatorname{Br}\left(\tau^{-} \rightarrow \nu_{\tau} \mathrm{K}_{1}(1270)\right)}{\operatorname{Br}\left(\tau^{-} \rightarrow \nu_{\tau} \mathrm{K}_{1}(1400)\right)+\operatorname{Br}\left(\tau^{-} \rightarrow \nu_{\tau} \mathrm{K}_{1}(1270)\right)}=0.71 \pm 0.16 \pm 0.11
$$

where the first uncertainty is due to the statistical uncertainty from the fit, and the second arises from the limited statistics of the Monte Carlo generated samples and the contribution from other systematic effects.

To determine if $R$ depends on assumptions made about the widths of the $\mathrm{K}_{1}$ resonances, the above procedure is repeated, simulating the signal portion of the distributions using $\tau^{-} \rightarrow \nu_{\tau} \mathrm{K}^{-} \pi^{-} \pi^{+}$Monte Carlo events generated through the $\mathrm{K}_{1}(1270)$ and $\mathrm{K}_{1}(1400)$ resonances, both with width $300 \mathrm{MeV}$. This procedure yields:

$$
R=0.68 \pm 0.13 \pm 0.11,
$$

which is in agreement with the central value of $R$ to within $0.2 \sigma$ of the combined Monte Carlo statistical uncertainty.

\section{$5.3 \quad \tau^{-} \rightarrow \nu_{\tau} \mathrm{K}^{-} \pi^{-} \mathrm{K}^{+}$}

The $\tau^{-} \rightarrow \nu_{\tau} \mathrm{K}^{-} \pi^{-} \mathrm{K}^{+}$candidate sample is expected to consist of $\tau^{-} \rightarrow \nu_{\tau} \pi^{-} \pi^{-} \pi^{+}\left(\pi^{0}\right)$ background, along with $\tau^{-} \rightarrow \nu_{\tau} \mathrm{K}^{-} \pi^{-} \mathrm{K}^{+}$signal, some $\tau^{-} \rightarrow \nu_{\tau} \mathrm{K}^{-} \pi^{-} \mathrm{K}^{+} \pi^{0}$ contamination, and some contamination from other tau decays and hadronic $\mathrm{Z}^{0}$ events.

To analyse the resonant structure of $\tau^{-} \rightarrow \nu_{\tau} \mathrm{K}^{-} \pi^{-} \mathrm{K}^{+}$decays, we examine the $M_{\mathrm{K}^{-} \pi^{-} \mathrm{K}^{+}}, M_{\mathrm{K}^{-} \mathrm{K}^{+}}$, and $M_{\pi^{-} \mathrm{K}^{+}}$invariant mass distributions of the candidates. To estimate the shape of the three-pion background in these distributions, we use the data $\tau^{-} \rightarrow \nu_{\tau} \pi^{-} \pi^{-} \pi^{+}\left(\pi^{0}\right)$ candidate sample, with the $5 \mathrm{GeV} / \mathrm{c}$ momentum selection placed on the particles which correspond to the two kaon candidates. This selection produces a sample of over 10000 events.

The shape of the $\tau^{-} \rightarrow \nu_{\tau} \mathrm{K}^{-} \pi^{-} \mathrm{K}^{+} \pi^{0}$ background in the distributions is parameterised with Monte Carlo $\tau^{-} \rightarrow \nu_{\tau} \mathrm{K}^{-} \pi^{-} \mathrm{K}^{+} \pi^{0}$ events generated through phase space. Other background fractions are expected to be on the order of a percent or less and are neglected. 
To describe the shape of the signal portion of these invariant mass distributions, we consider three different scenarios:

1. The $\tau^{-} \rightarrow \nu_{\tau} \mathrm{K}^{-} \pi^{-} \mathrm{K}^{+}$decays occur through the same intermediate resonant structure used in the default version of Tauola 2.4, as described in Section 3.

2. The $\tau^{-} \rightarrow \nu_{\tau} \mathrm{K}^{-} \pi^{-} \mathrm{K}^{+}$decays occur through $\mathrm{K}^{*}(892) \mathrm{K}^{-}$.

3. The $\tau^{-} \rightarrow \nu_{\tau} \mathrm{K}^{-} \pi^{-} \mathrm{K}^{+}$decays occur through phase space only.

Monte Carlo samples having at least 10 times the statistics of the expected signal component of the data $\tau^{-} \rightarrow \nu_{\tau} \mathrm{K}^{-} \pi^{-} \pi^{+}$candidates are generated under each of these resonant structure assumptions.

To determine the composition of the candidate sample, we divide the $M_{\mathrm{K}^{-} \pi^{-} \mathrm{K}^{+}}$, $M_{\mathrm{K}^{-} \mathrm{K}^{+}}$, and $M_{\pi^{-} \mathrm{K}^{+}}$invariant masses of the data, signal Monte Carlo, and background samples into 24 bins each. Further discrimination between signal and background is obtained by dividing the scalar sum of the momenta in each three-prong decay into 24 bins between 0 and $48 \mathrm{GeV} / \mathrm{c}$. Since this study is qualitative in nature only, correlations between the distributions are ignored.

The results of the fits using the three resonant structure assumptions are shown in Table 6. Table 6 also gives the $\chi^{2}$ per degree of freedom between the data and predicted $\sum P, M_{\mathrm{K}^{-} \pi^{-} \mathrm{K}^{+}}, M_{\mathrm{K}^{-} \mathrm{K}^{+}}$, and $M_{\pi^{-} \mathrm{K}^{+}}$distributions, where the normalisation of the predicted distributions is obtained from the fit results. The binning of these distributions is the same as that seen in Figure 7, which displays the results of the best fit to the data. A fit where the signal and $\tau^{-} \rightarrow \nu_{\tau} \mathrm{K}^{-} \pi^{-} \mathrm{K}^{+} \pi^{0}$ fractions are set to zero yields $-2 \log \mathcal{L} / \mathcal{L}_{\max }=51$. Since this fit has two fewer degrees of freedom than the first, the difference in $-2 \log \mathcal{L}$ should be $\chi^{2}$ distributed with two degrees of freedom if the sample does indeed consist of only three-pion background. This test disfavours this hypothesis at a confidence level of over $99 \%$.

Table 6 includes estimates of the efficiencies for $\tau^{-} \rightarrow \nu_{\tau} \mathrm{K}^{-} \pi^{-} \mathrm{K}^{+}$and $\tau^{-} \rightarrow \nu_{\tau} \mathrm{K}^{-} \pi^{-} \mathrm{K}^{+} \pi^{0}$ in the tau-pair sample to contribute to the $\tau^{-} \rightarrow \nu_{\tau} \mathrm{K}^{-} \pi^{-} \mathrm{K}^{+}$candidate samples. These efficiencies are not corrected for biases introduced by the $\tau^{-} \rightarrow \nu_{\tau} \mathrm{K}^{-} \pi^{-} \mathrm{K}^{+}$selection procedure. In order to verify that the signal fractions returned by the fit are reasonable, we use these efficiencies and the fit fractions to calculate estimates of the $\tau^{-} \rightarrow \nu_{\tau} \mathrm{K}^{-} \pi^{-} \mathrm{K}^{+}$and $\tau^{-} \rightarrow \nu_{\tau} \mathrm{K}^{-} \pi^{-} \mathrm{K}^{+} \pi^{0}$ branching ratios, which are also given in Table 6. Although the efficiencies have not been corrected for bias, these estimates are in agreement with the branching ratios obtained in Section 4 for all resonant structure assumptions.

As a cross-check, Monte Carlo generated $\tau^{-} \rightarrow \nu_{\tau}\left(\mathrm{K}^{*}(892) \mathrm{K} \pi\right)^{-}$decays are used to estimate the shape of the $\tau^{-} \rightarrow \nu_{\tau} \mathrm{K}^{-} \pi^{-} \mathrm{K}^{+} \pi^{0}$ in the data distributions, rather than 
$\tau^{-} \rightarrow \nu_{\tau} \mathrm{K}^{-} \pi^{-} \mathrm{K}^{+} \pi^{0}$ generated through non-resonant production. For all three assumptions made about the $\tau^{-} \rightarrow \nu_{\tau} \mathrm{K}^{-} \pi^{-} \mathrm{K}^{+}$intermediate resonant structure, these fits return signal fractions within $0.25 \sigma$ of the central values, and $\tau^{-} \rightarrow \nu_{\tau} \mathrm{K}^{-} \pi^{-} \mathrm{K}^{+} \pi^{0}$ fractions within $0.40 \sigma$ of the central values, where $\sigma$ refers to the statistical uncertainties of the central values of the fractions.

\section{Summary and Discussion}

From a sample of 295852 tau decays recorded in the OPAL detector between the years 1990 and 1995, we determine the branching ratios:

$$
\begin{aligned}
\operatorname{Br}\left(\tau^{-} \rightarrow \nu_{\tau} \mathrm{K}^{-} \pi^{-} \pi^{+}\left(\pi^{0}\right)\right) & =0.343 \pm 0.073 \pm 0.031 \% \\
\operatorname{Br}\left(\tau^{-} \rightarrow \nu_{\tau} \mathrm{K}^{-} \pi^{-} \mathrm{K}^{+}\left(\pi^{0}\right)\right) & =0.159 \pm 0.053 \pm 0.020 \% \\
\operatorname{Br}\left(\tau^{-} \rightarrow \nu_{\tau} \mathrm{K}^{-} \pi^{-} \pi^{+} \pi^{0}\right) & <0.179 \% \quad(95 \% \mathrm{CL}) \\
\operatorname{Br}\left(\tau^{-} \rightarrow \nu_{\tau} \mathrm{K}^{-} \pi^{-} \mathrm{K}^{+} \pi^{0}\right) & <0.247 \% \quad(95 \% \mathrm{CL}) \\
\operatorname{Br}\left(\tau^{-} \rightarrow \nu_{\tau} \mathrm{K}^{-} \pi^{-} \pi^{+}\right) & =0.360 \pm 0.082 \pm 0.048 \% \\
\operatorname{Br}\left(\tau^{-} \rightarrow \nu_{\tau} \mathrm{K}^{-} \pi^{-} \mathrm{K}^{+}\right) & =0.087 \pm 0.056 \pm 0.040 \%,
\end{aligned}
$$

where the first uncertainty is statistical and the second is systematic.

The $\tau^{-} \rightarrow \nu_{\tau} \mathrm{K}^{-} \pi^{-} \pi^{+}$inclusive and exclusive branching ratios are in agreement with both theory and previous empirical measurements, as listed in Table 1 .

In a separate analysis, we explore the resonant structure of $\tau^{-} \rightarrow \nu_{\tau} \mathrm{K}^{-} \pi^{-} \pi^{+}$and $\tau^{-} \rightarrow \nu_{\tau} \mathrm{K}^{-} \pi^{-} \mathrm{K}^{+}$decays. Under the assumption that the intermediate resonant structure of the tau decay to the $\nu_{\tau} \mathrm{K}^{-} \pi^{-} \pi^{+}$final state is dominated by the $\mathrm{K}_{1}$ intermediate resonances, we determine:

$$
R=\frac{\operatorname{Br}\left(\tau^{-} \rightarrow \nu_{\tau} \mathrm{K}_{1}(1270)\right)}{\operatorname{Br}\left(\tau^{-} \rightarrow \nu_{\tau} \mathrm{K}_{1}(1400)\right)+\operatorname{Br}\left(\tau^{-} \rightarrow \nu_{\tau} \mathrm{K}_{1}(1270)\right)}=0.71 \pm 0.16 \pm 0.11 .
$$

There are two previously published results for $R$ :

$$
\begin{array}{ll}
R=0.35_{-0.21}^{+0.29} & \text { TPC } / 2 \gamma 1994 \text { [3] } \\
R=0.91 \pm 0.29 & \text { ALEPH } 1999,19],
\end{array}
$$

with an average of $0.63 \pm 0.21$. The OPAL result is in agreement with this average. 
It has been suggested in [6] that theoretical predictions best match the world averages for the $\tau^{-} \rightarrow(\mathrm{K} \pi \pi)^{-}$branching ratios if the $\mathrm{K}_{1}$ resonances are in fact wider than the current world average widths, $\Gamma_{\mathrm{K}_{1}(1270)}=90 \mathrm{MeV}$ and $\Gamma_{\mathrm{K}_{1}(1400)}=174 \mathrm{MeV}$. From $S U(3)$ flavour symmetry arguments, reference [6] suggests that the actual widths of these resonances are likely to be approximately the $\mathrm{a}_{1}(1260)$ width $(250 \mathrm{MeV}$ or greater). We find that the data do indeed favour wider $K_{1}$ resonances, but that the world average widths are also consistent with the data.

\section{Acknowledgements}

We particularly wish to thank the SL Division for the efficient operation of the LEP accelerator at all energies and for their continuing close cooperation with our experimental group. We thank our colleagues from CEA, DAPNIA/SPP, CE-Saclay for their efforts over the years on the time-of-flight and trigger systems which we continue to use. In addition to the support staff at our own institutions we are pleased to acknowledge the

Department of Energy, USA, National Science Foundation, USA, Particle Physics and Astronomy Research Council, UK, Natural Sciences and Engineering Research Council, Canada, Israel Science Foundation, administered by the Israel Academy of Science and Humanities,

Minerva Gesellschaft, Benoziyo Center for High Energy Physics,

Japanese Ministry of Education, Science and Culture (the Monbusho) and a grant under the Monbusho International Science Research Program, Japanese Society for the Promotion of Science (JSPS), German Israeli Bi-national Science Foundation (GIF), Bundesministerium für Bildung, Wissenschaft, Forschung und Technologie, Germany, National Research Council of Canada, Research Corporation, USA, Hungarian Foundation for Scientific Research, OTKA T-029328, T023793 and OTKA F-023259.

\section{References}

[1] ALEPH Collaboration, R. Barate et al., Eur. Phys. J C1 (1998) 65.

[2] CLEO Collaboration, S. Richichi et al., CLNS 98/1573 (submitted to Phys. Rev. D).

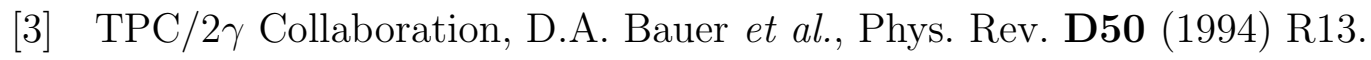


[4] DELCO Collaboration, G.B. Mills et al., Phys. Rev. Lett. 54 (1985) 624.

[5] M. Finkemeir and E. Mirkes, Zeit. Phys. C69 (1996) 243.

[6] E. Mirkes, Nucl. Phys. B (Proc. Suppl.), 55C (1997) 169.

[7] B.A. Li, Phys. Rev. D55 (1997) 1436.

[8] OPAL Collaboration, K. Ahmet et al., Nucl. Instr. and Meth. A305 (1991) 275.

[9] M. Hauschild et al., Nucl. Instr. and Meth. A314 (1992) 74.

[10] S. Jadach, B.F.L. Ward and Z. Was, Comp. Phys. Comm. 79 (1994) 503.

[11] S. Jadach, Z. Was, R. Decker and J.H. Kuhn, Comp. Phys. Comm. 76 (1993) 361.

[12] J. Allison et al., Nucl. Instr. and Meth. A317 (1992) 47.

[13] M. Suzuki, Phys. Rev. D47 (1993) 1252.

[14] Review of Particle Physics, Eur. Phys. J. C3 (1998) 1.

[15] D.M. Manley and E.M. Saleski, Phys. Rev. D45 (1992) 4002.

[16] ACCMOR Collaboration, C. Daum et al., Nucl. Phys. B187 (1981) 1.

[17] OPAL Collaboration, K. Ackerstaff et al., Eur. Phys. J. C4 (1998) 193.

[18] OPAL Collaboration, K. Ackerstaff et al., Zeit. Phys. C68 (1995) 555.

[19] ALEPH Collaboration, R. Barate et al., CERN-EP/99-026 (submitted to Eur. Phys. J. C). 


\begin{tabular}{|c|c|c|c|c|}
\hline $\begin{array}{l}\tau^{-} \text {DECAY } \\
\text { MODE }\end{array}$ & Strangeness & \multicolumn{2}{|c|}{$\begin{array}{c}\text { EXPERIMENT } \\
\text { BR }(\%) \\
\end{array}$} & $\begin{array}{c}\text { THEORY } \\
\text { BR }(\%)\end{array}$ \\
\hline$\nu_{\tau} \mathrm{K}^{-} \pi^{-} \pi^{+}\left(\pi^{0}\right)$ & -1 & $\begin{array}{l}0.275 \pm 0.064 \\
0.421 \pm 0.068 \\
0.58_{-0.18}^{+0.19} \\
0.22_{-0.16}^{+0.17} \\
0.343 \pm 0.079\end{array}$ & 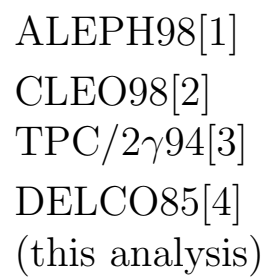 & - \\
\hline$\nu_{\tau} \mathrm{K}^{-} \pi^{-} \mathrm{K}^{+}\left(\pi^{0}\right)$ & 0 & $\begin{array}{l}0.238 \pm 0.042 \\
0.178 \pm 0.036 \\
0.15_{-0.08}^{+0.09} \\
0.159 \pm 0.057\end{array}$ & $\begin{array}{l}\text { ALEPH98[1] } \\
\text { CLEO98[2] } \\
\text { TPC } / 2 \gamma 94[3] \\
\text { (this analysis) }\end{array}$ & - \\
\hline$\nu_{\tau} \mathrm{K}^{-} \pi^{-} \pi^{+}$ & -1 & $\begin{array}{l}0.214 \pm 0.047 \\
0.346 \pm 0.061 \\
0.360 \pm 0.095\end{array}$ & $\begin{array}{l}\text { ALEPH98 1] } \\
\text { CLEO98 2] } \\
\text { (this analysis) }\end{array}$ & $\begin{array}{l}0.77 \text { [5] } \\
0.35 \text { to } 0.45 \text { [6] } \\
0.18 \text { [7] }\end{array}$ \\
\hline$\nu_{\tau} \mathrm{K}^{-} \pi^{-} \mathrm{K}^{+}$ & 0 & $\begin{array}{l}0.163 \pm 0.027 \\
0.145 \pm 0.031 \\
0.22_{-0.12}^{+0.18} \\
0.087 \pm 0.069\end{array}$ & $\begin{array}{l}\text { ALEPH98 1] } \\
\text { CLEO98 2] } \\
\text { DELCO85 } 4] \\
\text { (this analysis) }\end{array}$ & $\begin{array}{l}0.20 \text { [5] } \\
0.26 \text { [0] }\end{array}$ \\
\hline$\nu_{\tau} \mathrm{K}^{-} \pi^{-} \pi^{+} \pi^{0}$ & -1 & $\begin{array}{l}0.061 \pm 0.043 \\
0.075 \pm 0.032 \\
<0.179\end{array}$ & $\begin{array}{l}\text { ALEPH98 [1] } \\
\text { CLEO98[2] } \\
\text { (this analysis) }\end{array}$ & - \\
\hline$\nu_{\tau} \mathrm{K}^{-} \pi^{-} \mathrm{K}^{+} \pi^{0}$ & 0 & $\begin{array}{l}0.075 \pm 0.033 \\
0.033 \pm 0.019 \\
<0.247\end{array}$ & $\begin{array}{l}\text { ALEPH98 [1] } \\
\text { CLEO98 2 } \\
\text { (this analysis) }\end{array}$ & - \\
\hline$\nu_{\tau} \pi^{-} \pi^{-} \mathrm{K}^{+}\left(\pi^{0}\right)$ & +1 & $<0.25$ & $\mathrm{TPC} / 2 \gamma 94 \sqrt[3]{3}$ & - \\
\hline$\nu_{\tau} \mathrm{K}^{-} \mathrm{K}^{-} \pi^{+}\left(\pi^{0}\right)$ & -2 & $<0.09$ & $\mathrm{TPC} / 2 \gamma 94[3]$ & - \\
\hline$\nu_{\tau} \mathrm{K}^{-} \mathrm{K}^{-} \mathrm{K}^{+}\left(\pi^{0}\right)$ & -1 & $\begin{array}{l}<0.21 \\
<0.019\end{array}$ & $\begin{array}{l}\mathrm{TPC} / 2 \gamma 94[3] \\
\text { ALEPH98[1] }\end{array}$ & - \\
\hline
\end{tabular}

Table 1: Three-prong decays of the tau lepton which include charged kaons in the final state (charge conjugate decays are implied). Experimental uncertainties are the combined statistical and systematic uncertainties. Branching ratios quoted as limits are the $95 \%$ confidence limits, and the $\left(\pi^{0}\right)$ notation refers to decay modes with or without an accompanying $\pi^{0}$. 


\begin{tabular}{|c|c|c|c|c|c|c|}
\hline & \multicolumn{3}{|c|}{$\pi^{0}$-depleted Sample } & \multicolumn{3}{|c|}{$\pi^{0}$-enhanced Sample } \\
\hline & \multirow[t]{2}{*}{ like-sign } & \multicolumn{2}{|c|}{ unlike-sign } & \multirow[t]{2}{*}{ like-sign } & \multicolumn{2}{|c|}{ unlike-sign } \\
\hline & & $\begin{array}{l}\mathrm{K}^{-} \pi^{-} \mathrm{K}^{+} \\
\text {enhanced }\end{array}$ & $\begin{array}{r}\mathrm{K}^{-} \pi^{-} \mathrm{K}^{+} \\
\text {depleted }\end{array}$ & & $\begin{array}{l}\mathrm{K}^{-} \pi^{-} \mathrm{K}^{+} \\
\text {enhanced }\end{array}$ & $\begin{array}{r}\mathrm{K}^{-} \pi^{-} \mathrm{K}^{+} \\
\text {depleted }\end{array}$ \\
\hline$N_{\mathrm{K}}$ & $128.4 \pm 16.5 \pm 2.3$ & $36.7 \pm 9.5 \pm 1.2$ & $1.4 \pm 3.7 \pm 0.5$ & $29.5 \pm 9.2 \pm 1.1$ & $5.9 \pm 5.6 \pm 0.6$ & $4.6 \pm 3.7 \pm 0.3$ \\
\hline$N_{\text {bkgnd }}$ & $5.8 \pm 1.8$ & 0 & 0 & $8.5 \pm 2.1$ & $2.5 \pm 1.5$ & 0 \\
\hline$\epsilon_{\mathrm{K}^{-} \pi^{-} \pi^{+}}$ & $0.094 \pm 0.003$ & $<0.001$ & $<0.001$ & $0.013 \pm 0.001$ & $<0.001$ & $<0.001$ \\
\hline$\epsilon_{\mathrm{K}^{-} \pi^{-} \mathrm{K}^{+}}$ & $0.078 \pm 0.003$ & $0.104 \pm 0.003$ & $0.003 \pm 0.001$ & $0.007 \pm 0.001$ & $0.008 \pm 0.001$ & $<0.001$ \\
\hline$\epsilon_{\pi^{-} \pi^{-} \mathrm{K}^{+}}$ & $<0.001$ & $0.065 \pm 0.006$ & $0.029 \pm 0.002$ & $<0.001$ & $0.008 \pm 0.001$ & $0.005 \pm 0.001$ \\
\hline$\epsilon_{\mathrm{K}^{-} \pi^{-} \pi^{+} \pi^{0}}$ & $0.044 \pm 0.002$ & $<0.001$ & $<0.001$ & $0.047 \pm 0.002$ & $<0.001$ & $<0.001$ \\
\hline$\epsilon_{\mathrm{K}^{-} \pi^{-}} \mathrm{K}^{+} \pi^{0}$ & $0.048 \pm 0.003$ & $0.053 \pm 0.003$ & $0.001 \pm 0.001$ & $0.030 \pm 0.002$ & $0.032 \pm 0.002$ & $<0.001$ \\
\hline$\epsilon_{\pi^{-} \pi^{-} \mathrm{K}^{+} \pi^{0}}$ & $<0.001$ & $0.033 \pm 0.002$ & $0.012 \pm 0.001$ & $<0.001$ & $0.036 \pm 0.001$ & $0.011 \pm 0.001$ \\
\hline
\end{tabular}

Table 2: The number of kaons in each data sample, $N_{\mathrm{K}}$, where the first uncertainty is the statistical uncertainty from the maximum likelihood fit to the measured $\mathrm{d} E / \mathrm{d} x$ of the tracks in the sample, and the second is the systematic uncertainty arising from the uncertainties in the parameterisation of $\mathrm{d} E / \mathrm{d} x$. Also shown are the estimated backgrounds and the average efficiencies for the various signal channels which contribute to each sample. 


\begin{tabular}{|l|r|r||r|r||r|r|}
\hline & \multicolumn{6}{|c|}{ Branching Ratios (\%) } \\
\cline { 2 - 6 } & $\mathrm{B}_{\mathrm{K}^{-} \pi^{-} \pi^{+}\left(\pi^{0}\right)}$ & $\mathrm{B}_{\mathrm{K}^{-} \pi^{-} \mathrm{K}^{+}\left(\pi^{0}\right)}$ & $\mathrm{B}_{\mathrm{K}^{-} \pi^{-} \pi^{+} \pi^{0}}$ & $\mathrm{~B}_{\mathrm{K}^{-} \pi^{-} \mathrm{K}^{+} \pi^{0}}$ & $\mathrm{~B}_{\mathrm{K}^{-} \pi^{-} \pi^{+}}$ & $\mathrm{B}_{\mathrm{K}^{-} \pi^{-} \mathrm{K}^{+}}$ \\
\hline Central value & 0.343 & 0.159 & -0.017 & 0.072 & 0.360 & 0.087 \\
\hline$\sigma$ (stat) & \pm 0.073 & \pm 0.053 & \pm 0.076 & \pm 0.085 & \pm 0.082 & \pm 0.056 \\
$\sigma$ (dE/d $x$ sys) & \pm 0.017 & \pm 0.010 & \pm 0.020 & \pm 0.015 & \pm 0.013 & \pm 0.016 \\
$\sigma$ (MC stat) & \pm 0.023 & \pm 0.012 & \pm 0.029 & \pm 0.018 & \pm 0.029 & \pm 0.018 \\
$\sigma$ ( $\sum E / \sum P$ sys) & \pm 0.012 & \pm 0.012 & \pm 0.049 & \pm 0.045 & \pm 0.036 & \pm 0.032 \\
\hline
\end{tabular}

Table 3: Summary of the branching ratio central values and sources of uncertainty. 


\begin{tabular}{|l|cccc|}
\hline & $\mathrm{B}_{\mathrm{K}^{-} \pi^{-} \pi^{+}}$ & $\mathrm{B}_{\mathrm{K}^{-} \pi^{-} \mathrm{K}^{+}}$ & $\mathrm{B}_{\mathrm{K}^{-} \pi^{-} \pi^{+} \pi^{0}}$ & $\mathrm{~B}_{\mathrm{K}^{-} \pi^{-} \mathrm{K}^{+} \pi^{0}}$ \\
\hline $\mathrm{B}_{\mathrm{K}^{-} \pi^{-} \pi^{+}}$ & +1.0 & -0.3 & -0.6 & +0.1 \\
$\mathrm{~B}_{\mathrm{K}^{-} \pi^{-} \mathrm{K}^{+}}$ & -0.3 & +1.0 & +0.4 & -0.8 \\
$\mathrm{~B}_{\mathrm{K}^{-} \pi^{-} \pi^{+} \pi^{0}}$ & -0.6 & +0.3 & +1.0 & -0.5 \\
$\mathrm{~B}_{\mathrm{K}^{-} \pi^{-} \mathrm{K}^{+} \pi^{0}}$ & +0.1 & -0.8 & -0.5 & +1.0 \\
\hline
\end{tabular}

Table 4: Correlations between the exclusive branching ratios. 


\begin{tabular}{|c|c|c|c|c|}
\hline & $\begin{array}{c}\mathrm{K}_{1}(1270) \\
\text { and } \mathrm{K}_{1}(1400) \\
\Gamma_{1270}=90 \mathrm{MeV} \\
\Gamma_{1400}=174 \mathrm{MeV} \\
\end{array}$ & $\begin{array}{c}\mathrm{K}_{1}(1270) \\
\text { and } \mathrm{K}_{1}(1400) \\
\Gamma_{1270}=300 \mathrm{MeV} \\
\Gamma_{1400}=300 \mathrm{MeV}\end{array}$ & $\begin{array}{c}\mathrm{K}^{*}(892) \pi^{-} \\
\text {and } \rho(770) K^{-}\end{array}$ & Phase Space \\
\hline $\begin{array}{l}f_{\text {signal } \# 1} \\
f_{\text {signal } \# 2} \\
f_{\mathrm{K}^{-} \pi^{-}-\pi^{+} \pi^{0}} \\
-2 \log \mathcal{L} / \mathcal{L}_{\max }\end{array}$ & $\begin{array}{l}f_{\mathrm{K}_{1}(1400)}=0.043 \pm 0.025 \pm 0.017 \\
f_{\mathrm{K}_{1}(1270)}=0.096 \pm 0.034 \pm 0.028 \\
0.066 \pm 0.028 \\
8\end{array}$ & $\begin{array}{l}f_{\mathrm{K}_{1}(1400)}=0.065 \pm 0.025 \pm 0.021 \\
f_{\mathrm{K}_{1}(1270)}=0.121 \pm 0.039 \pm 0.036 \\
0.048 \pm 0.036 \\
0\end{array}$ & $\begin{array}{l}f_{\mathrm{K}^{*} \pi}=0.100 \pm 0.027 \\
f_{\rho \mathrm{K}}=0.040 \pm 0.235 \\
0.076 \pm 0.030 \\
3\end{array}$ & $\begin{array}{l}0.029 \pm 0.033 \\
- \\
0.082 \pm 0.029 \\
96\end{array}$ \\
\hline $\begin{array}{ll}\chi^{2} & \left(\sum P\right) \\
\chi^{2} & \left(M_{\mathrm{K}^{-} \pi^{-} \pi^{+}}\right) \\
\chi^{2} & \left(M_{\mathrm{K}^{-} \pi^{+}}\right) \\
\chi^{2} & \left(M_{\pi^{-} \pi^{+}}\right) \\
\end{array}$ & $\begin{array}{l}15.9 / 18 \\
26.5 / 20 \\
22.3 / 17 \\
26.1 / 18 \\
\end{array}$ & $\begin{array}{l}17.4 / 18 \\
23.7 / 20 \\
19.5 / 17 \\
27.8 / 18 \\
\end{array}$ & $\begin{array}{l}17.3 / 18 \\
26.7 / 20 \\
21.2 / 17 \\
26.3 / 18 \\
\end{array}$ & $\begin{array}{l}22.9 / 18 \\
29.0 / 20 \\
37.0 / 17 \\
25.9 / 18 \\
\end{array}$ \\
\hline $\begin{array}{l}\epsilon_{\mathrm{K}^{-} \pi^{-} \pi^{+}} \\
\epsilon_{\mathrm{K}^{-} \pi^{-} \pi^{+} \pi^{0}}\end{array}$ & $\begin{array}{l}0.22 \\
0.17 \\
\end{array}$ & $\begin{array}{l}0.22 \\
0.17 \\
\end{array}$ & $\begin{array}{l}0.22 \\
0.17 \\
\end{array}$ & $\begin{array}{l}0.21 \\
0.17 \\
\end{array}$ \\
\hline $\begin{array}{l}\mathrm{B}_{\mathrm{K}^{-} \pi^{-} \pi^{+}}(\%) \\
\mathrm{B}_{\mathrm{K}^{-} \pi^{-} \pi^{+} \pi^{0}}(\%)\end{array}$ & $\begin{array}{l}0.207 \pm 0.054 \\
0.128 \pm 0.054\end{array}$ & $\begin{array}{l}0.271 \pm 0.058 \\
0.093 \pm 0.069\end{array}$ & $\begin{array}{l}0.205 \pm 0.334 \\
0.147 \pm 0.058\end{array}$ & $\begin{array}{l}0.045 \pm 0.052 \\
0.158 \pm 0.056\end{array}$ \\
\hline
\end{tabular}

Table 5: Summary of the $\tau^{-} \rightarrow \nu_{\tau} \mathrm{K}^{-} \pi^{-} \pi^{+}$signal and $\tau^{-} \rightarrow \nu_{\tau} \mathrm{K}^{-} \pi^{-} \pi^{+} \pi^{0}$ background fractions obtained from the binned maximum likelihood fits to the data $\tau^{-} \rightarrow \nu_{\tau} \mathrm{K}^{-} \pi^{-} \pi^{+}$candidate invariant mass and $\sum P$ distributions. The first uncertainties are the statistical uncertainties from the fits. The second uncertainties for $f_{\mathrm{K}_{1}(1400)}$ and $f_{\mathrm{K}_{1}(1270)}$ are the systematic uncertainties due to the limited statistics in the Monte Carlo generated samples. Also shown are the values of $-2 \log \mathcal{L} / \mathcal{L}_{\max }$ from the fit (relative to the best fit), and the resulting $\chi^{2}$ per degree of freedom between the various data and predicted distributions. Estimates of the efficiencies for signal and $\tau^{-} \rightarrow \nu_{\tau} \mathrm{K}^{-} \pi^{-} \pi^{+} \pi^{0}$ decays in the tau-pair candidate sample to contribute to the $\tau^{-} \rightarrow \nu_{\tau} \mathrm{K}^{-} \pi^{-} \pi^{+}$sample are given. From these efficiencies and the fraction estimates from the fits, estimates of the branching ratios are calculated. These branching ratios have not been corrected for biases introduced by the event selection and are used as a cross-check of the analysis only. The branching ratio uncertainties are from the statistical uncertainties of the fit fractions. 


\begin{tabular}{|l|l|l|l|}
\hline & $\begin{array}{c}\text { Tauola 2.4 default } \\
\text { mix of } a_{1}(1260) \\
\text { and } \rho(1700)\end{array}$ & $\mathrm{K}^{*}(892) \mathrm{K}^{-}$ & Phase Space \\
\hline$f_{\mathrm{K}^{-} \pi^{-} \mathrm{K}^{+}}$ & $0.473 \pm 0.057$ & $0.447 \pm 0.074$ & $0.517 \pm 0.086$ \\
$f_{\mathrm{K}^{-} \pi^{-} \mathrm{K}^{+} \pi^{0}}$ & $0.000 \pm 0.304$ & $0.141 \pm 0.059$ & $0.109 \pm 0.061$ \\
$-2 \log \mathcal{L} / \mathcal{L}_{\max }$ & 0 & 18 & 21 \\
\hline$\chi^{2}\left(\sum P\right)$ & $7.6 / 5$ & $7.3 / 5$ & $8.4 / 5$ \\
$\chi^{2}\left(M_{\mathrm{K}^{-} \pi^{-} \mathrm{K}^{+}}\right)$ & $7.0 / 6$ & $8.7 / 6$ & $7.8 / 6$ \\
$\chi^{2}\left(M_{\left.\mathrm{K}^{-} \mathrm{K}^{+}\right)}\right)$ & $5.5 / 6$ & $10.6 / 6$ & $8.5 / 6$ \\
$\chi^{2}\left(M_{\pi^{-} \mathrm{K}^{+}}\right)$ & $3.8 / 5$ & $4.5 / 5$ & $8.6 / 5$ \\
\hline$\epsilon_{\mathrm{K}^{-} \pi^{-} \mathrm{K}^{+}}$ & 0.13 & 0.14 & 0.15 \\
$\epsilon_{\mathrm{K}^{-} \pi^{-} \mathrm{K}^{+} \pi^{0}}$ & 0.10 & 0.10 & 0.10 \\
\hline $\mathrm{B}_{\mathrm{K}^{-} \pi^{-} \mathrm{K}^{+}}(\%)$ & $0.096 \pm 0.012$ & $0.085 \pm 0.014$ & $0.096 \pm 0.016$ \\
$\mathrm{~B}_{\mathrm{K}^{-} \pi^{-} \mathrm{K}^{+} \pi^{0}}(\%)$ & $0.000 \pm 0.079$ & $0.037 \pm 0.015$ & $0.028 \pm 0.016$ \\
\hline
\end{tabular}

Table 6: Summary of the $\tau^{-} \rightarrow \nu_{\tau} \mathrm{K}^{-} \pi^{-} \mathrm{K}^{+}$signal and $\tau^{-} \rightarrow \nu_{\tau} \mathrm{K}^{-} \pi^{-} \mathrm{K}^{+} \pi^{0}$ background fractions obtained from the binned maximum likelihood fits to the data $\tau^{-} \rightarrow \nu_{\tau} \mathrm{K}^{-} \pi^{-} \mathrm{K}^{+}$candidate invariant mass and $\sum P$ distributions. The uncertainties are the statistical uncertainties from the fits. Also shown are the values of $-2 \log \mathcal{L} / \mathcal{L}_{\text {max }}$ from the fit (relative to the best fit), and the resulting $\chi^{2}$ per degree of freedom between the various data and predicted distributions. Estimates of the efficiencies for signal and $\tau^{-} \rightarrow \nu_{\tau} \mathrm{K}^{-} \pi^{-} \mathrm{K}^{+} \pi^{0}$ decays in the tau-pair candidate sample to contribute to the $\tau^{-} \rightarrow \nu_{\tau} \mathrm{K}^{-} \pi^{-} \mathrm{K}^{+}$sample are given. From these efficiencies and the fraction estimates from the fits, estimates of the branching ratios are calculated. These branching ratios have not been corrected for biases introduced by the event selection and are used as a cross-check of the analysis only. The branching ratio uncertainties are from the statistical uncertainties of the fit fractions. 
OPAL
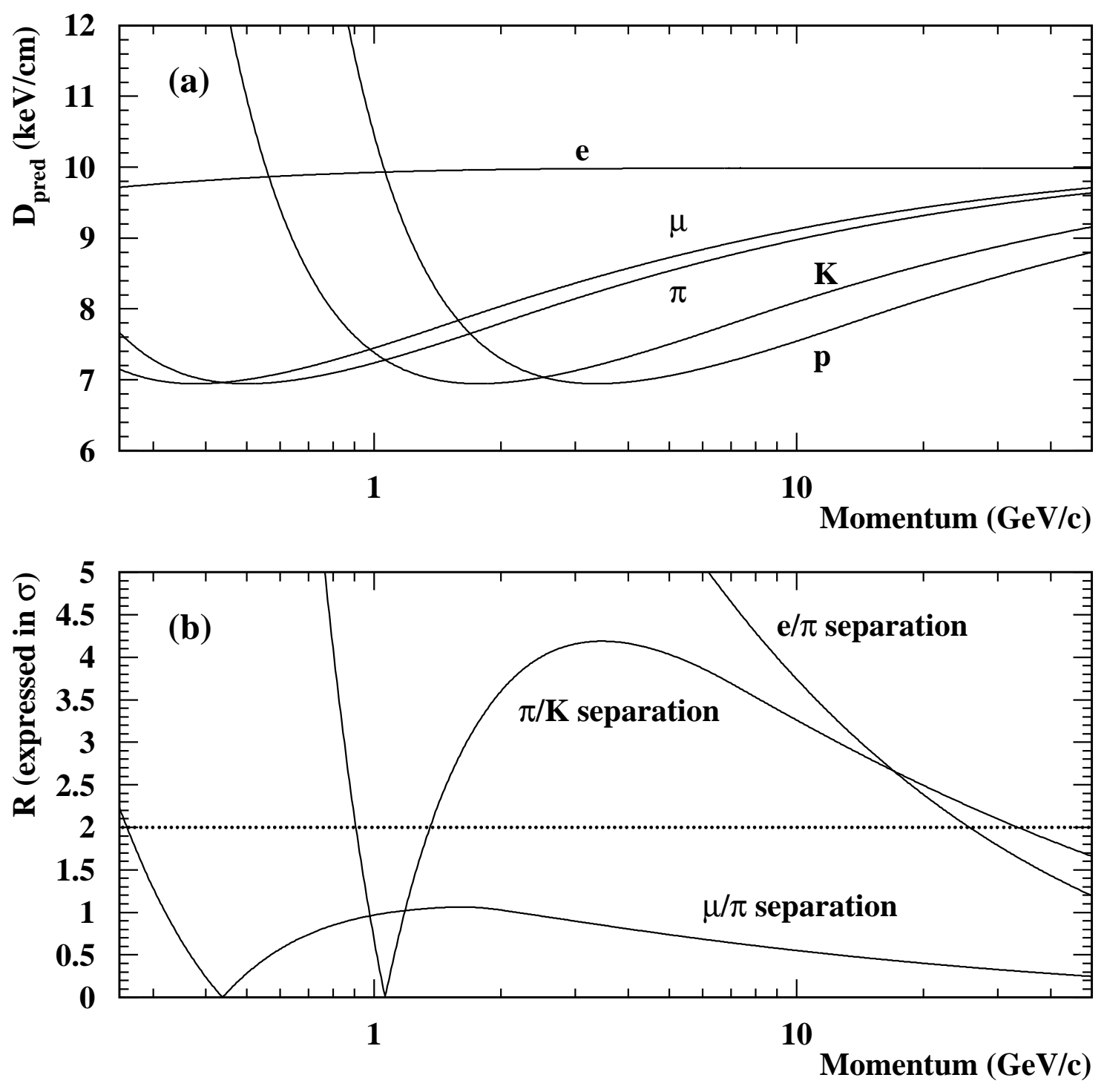

Figure 1: (a) shows the ionisation energy loss $D_{\text {pred }}$ as a function of the momentum for various particles in the OPAL jet chamber. (b) shows the resolution power $\mathcal{R}_{i j}$ expressed in terms of the $\mathrm{d} E / \mathrm{d} x$ resolution $\sigma$, for various pairs of particle species $i$ and $j$. 
OPAL
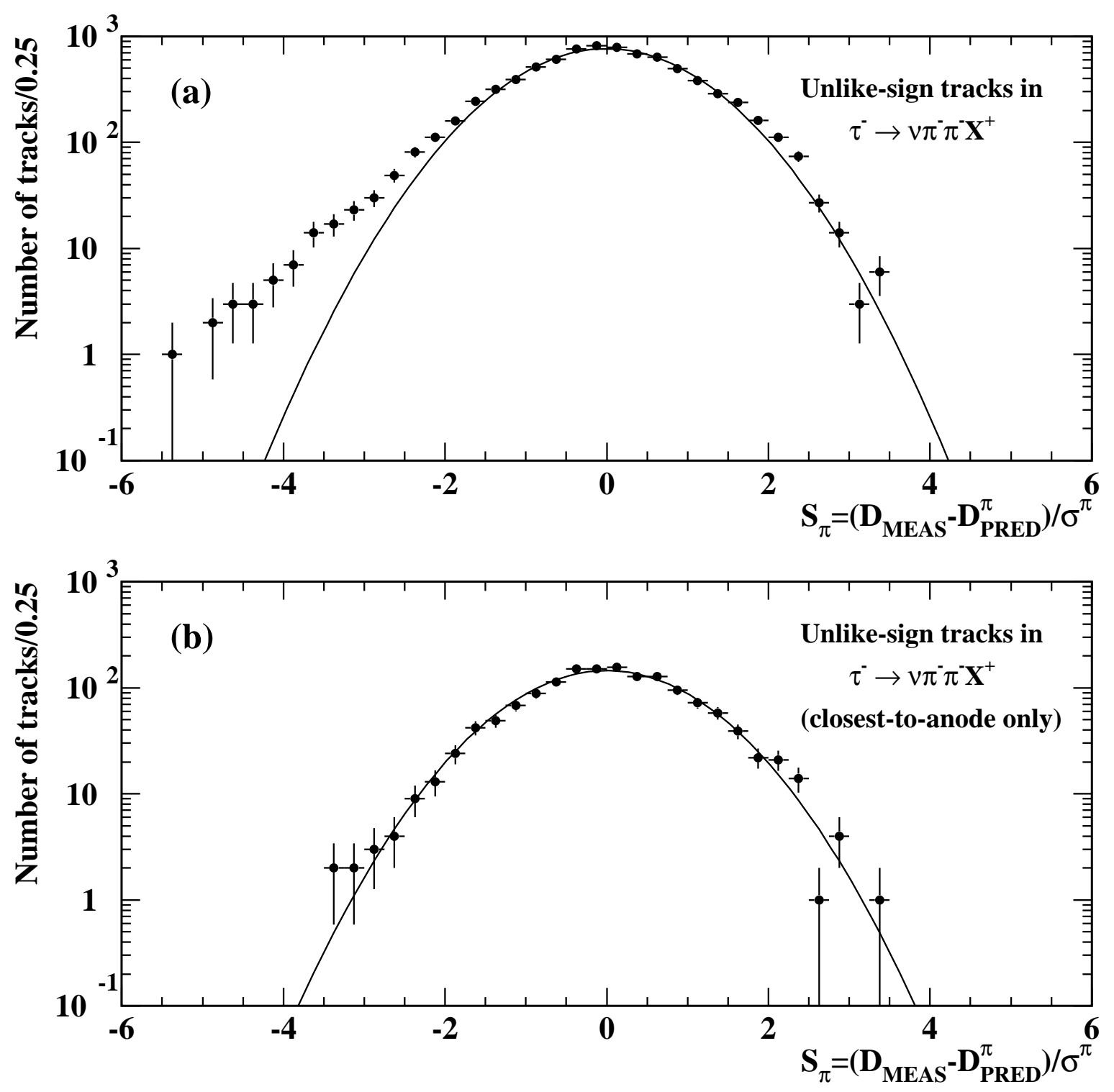

Figure 2: (a) is the stretch $\mathrm{d} E / \mathrm{d} x$ distribution under the pion hypothesis for the unlike-sign tracks in the data $\tau^{-} \rightarrow \nu_{\tau} \pi^{-} \pi^{-} \mathrm{X}^{+}\left(\pi^{0}\right)$ candidate sample (points). This sample is expected to have less than three kaons present. The curve is a unit width Gaussian of zero mean, whose normalisation is fit to the central part of the distribution. A significant non-Gaussian component is evident. (b) is the same distribution for the unlike-sign tracks in the data first-track $\tau^{-} \rightarrow \nu_{\tau} \pi^{-} \pi^{-} \mathrm{X}^{+}\left(\pi^{0}\right)$ candidate sample. 
OPAL
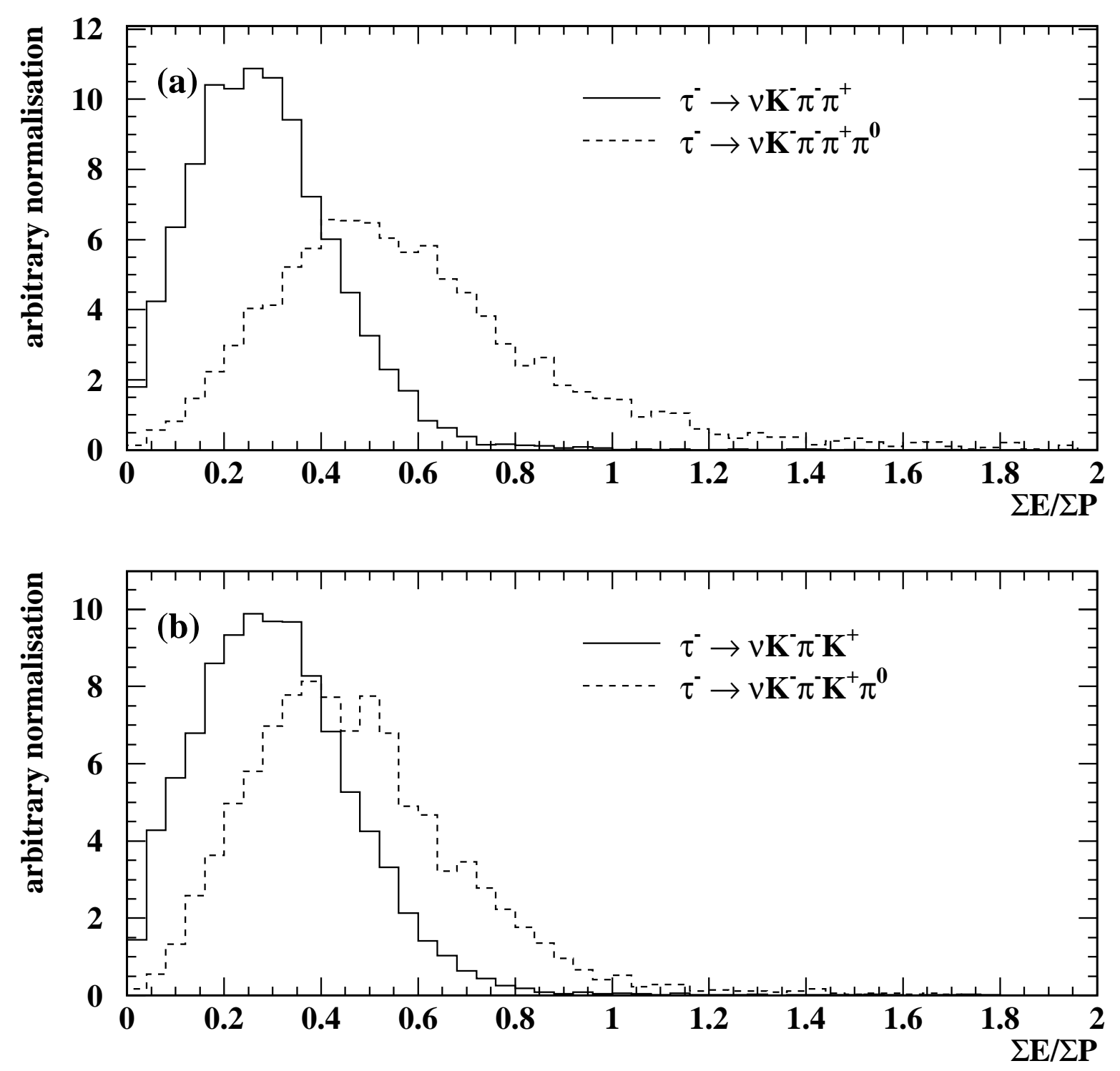

Figure 3: (a) shows the $\sum E / \sum P$ distributions for Monte Carlo generated $\tau^{-} \rightarrow \nu_{\tau} \mathrm{K}^{-} \pi^{-} \pi^{+}$and $\tau^{-} \rightarrow \nu_{\tau} \mathrm{K}^{-} \pi^{-} \pi^{+} \pi^{0}$. (b) shows the same distributions for Monte Carlo generated $\tau^{-} \rightarrow \nu_{\tau} \mathrm{K}^{-} \pi^{-} \mathrm{K}^{+}$and $\tau^{-} \rightarrow \nu_{\tau} \mathrm{K}^{-} \pi^{-} \mathrm{K}^{+} \pi^{0}$. 


\section{OPAL}
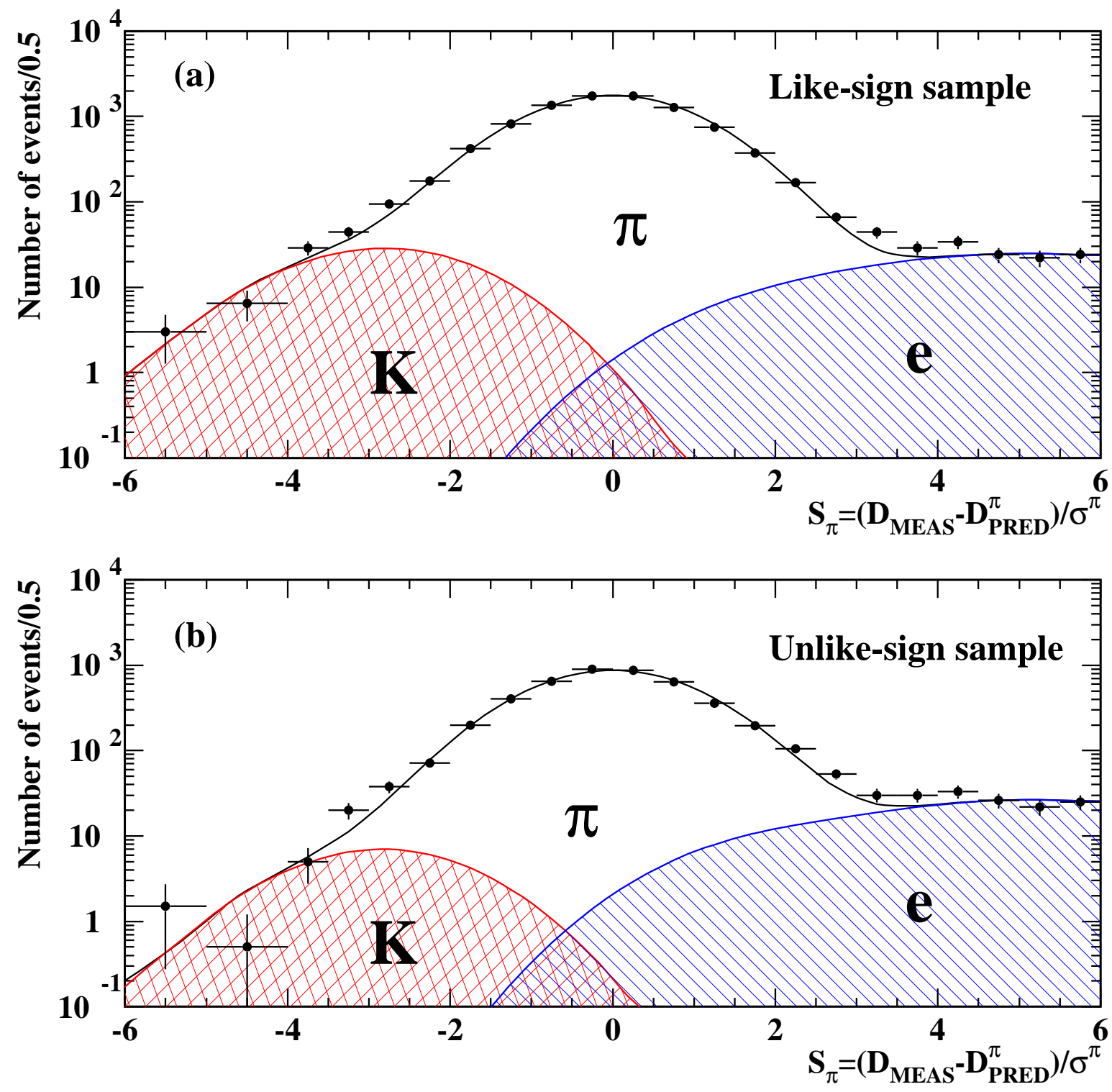

Figure 4: (a) shows the stretch $\mathrm{d} E / \mathrm{d} x$ distribution of tracks in the data inclusive like-sign sample (points). The overlaid curves are the predicted distributions for the kaons, pions and electrons in the sample, assuming a Gaussian resolution function for the $\mathrm{d} E / \mathrm{d} x$ measurements. The normalisation of the curves comes from the results of the likelihood fit. (b) shows the same distribution for tracks in the data inclusive unlike-sign sample. 
OPAL
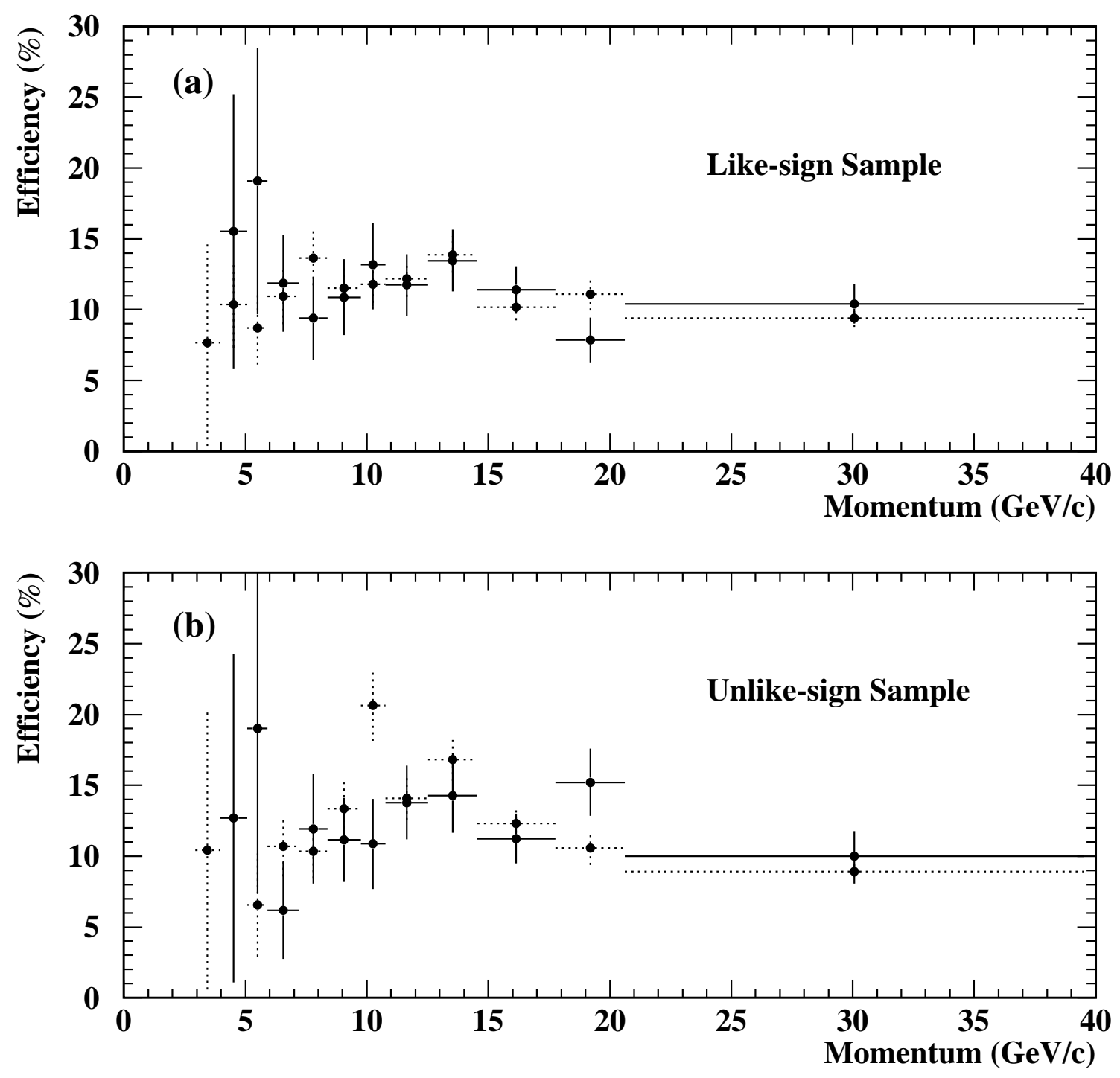

Figure 5: (a) shows the efficiency as a function of kaon momentum for $\tau^{-} \rightarrow \nu_{\tau} \mathrm{K}^{-} \pi^{-} \pi^{+}$ in the pre-selected tau-pair sample to contribute kaons to the like-sign sample; the solid points represent the default Tauola 2.4 generation of $\tau^{-} \rightarrow \nu_{\tau} \mathrm{K}^{-} \pi^{-} \pi^{+}$and the dotted points are calculated using $\tau^{-} \rightarrow \nu_{\tau} \mathrm{K}^{-} \pi^{-} \pi^{+}$generated through phase space only. (b) shows the efficiency versus kaon momentum for $\tau^{-} \rightarrow \nu_{\tau} \mathrm{K}^{-} \pi^{-} \mathrm{K}^{+}$in the pre-selected tau-pair sample to contribute kaons to the unlike-sign sample; the solid points represent the default Tauola 2.4 generation of $\tau^{-} \rightarrow \nu_{\tau} \mathrm{K}^{-} \pi^{-} \mathrm{K}^{+}$and the dotted points are calculated using $\tau^{-} \rightarrow \nu_{\tau} \mathrm{K}^{-} \pi^{-} \mathrm{K}^{+}$generated through phase space only. The branching ratio central values are calculated using $\tau^{-} \rightarrow \nu_{\tau} \mathrm{K}^{-} \pi^{-} \pi^{+}$and $\tau^{-} \rightarrow \nu_{\tau} \mathrm{K}^{-} \pi^{-} \mathrm{K}^{+}$efficiencies estimated with the default version of Tauola 2.4. 
OPAL $\tau^{-} \rightarrow v \mathbf{K}^{-} \pi^{-} \pi^{+}$candidates
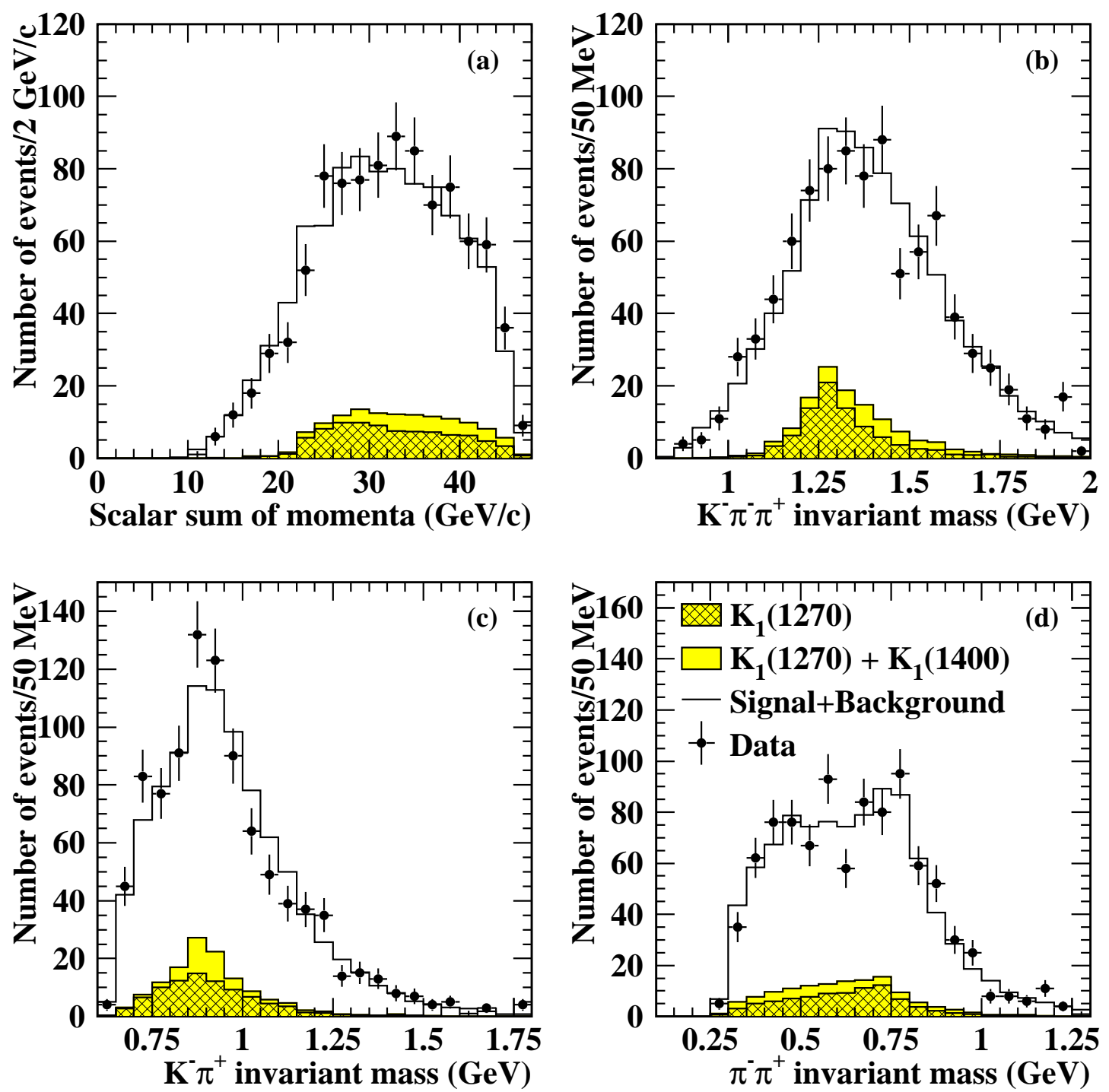

Figure 6: (a) is the $\sum P$ distribution and (b), (c), and (d) are the invariant mass distributions of the data $\tau^{-} \rightarrow \nu_{\tau} \mathrm{K}^{-} \pi^{-} \pi^{+}$candidate sample (points). The histogram represents the predicted distribution, in which the normalisation of the background and signal components comes from the best fit results of the binned maximum likelihood fit which took the correlations between the invariant mass distributions into account. The shape of the signal is estimated using $\tau^{-} \rightarrow \nu_{\tau} \mathrm{K}^{-} \pi^{-} \pi^{+}$events generated through the $K_{1}(1270)$ and the $K_{1}(1400)$ resonances, where the widths of the resonances are taken to be 90 and $174 \mathrm{MeV}$, respectively [14]. The shape of the $\tau^{-} \rightarrow \nu_{\tau} \pi^{-} \pi^{-} \pi^{+}\left(\pi^{0}\right)$ background in the distributions is obtained from a data control sample. 
OPAL $\tau^{-} \rightarrow v \mathbf{K}^{-} \pi^{-} \mathbf{K}^{+}$candidates
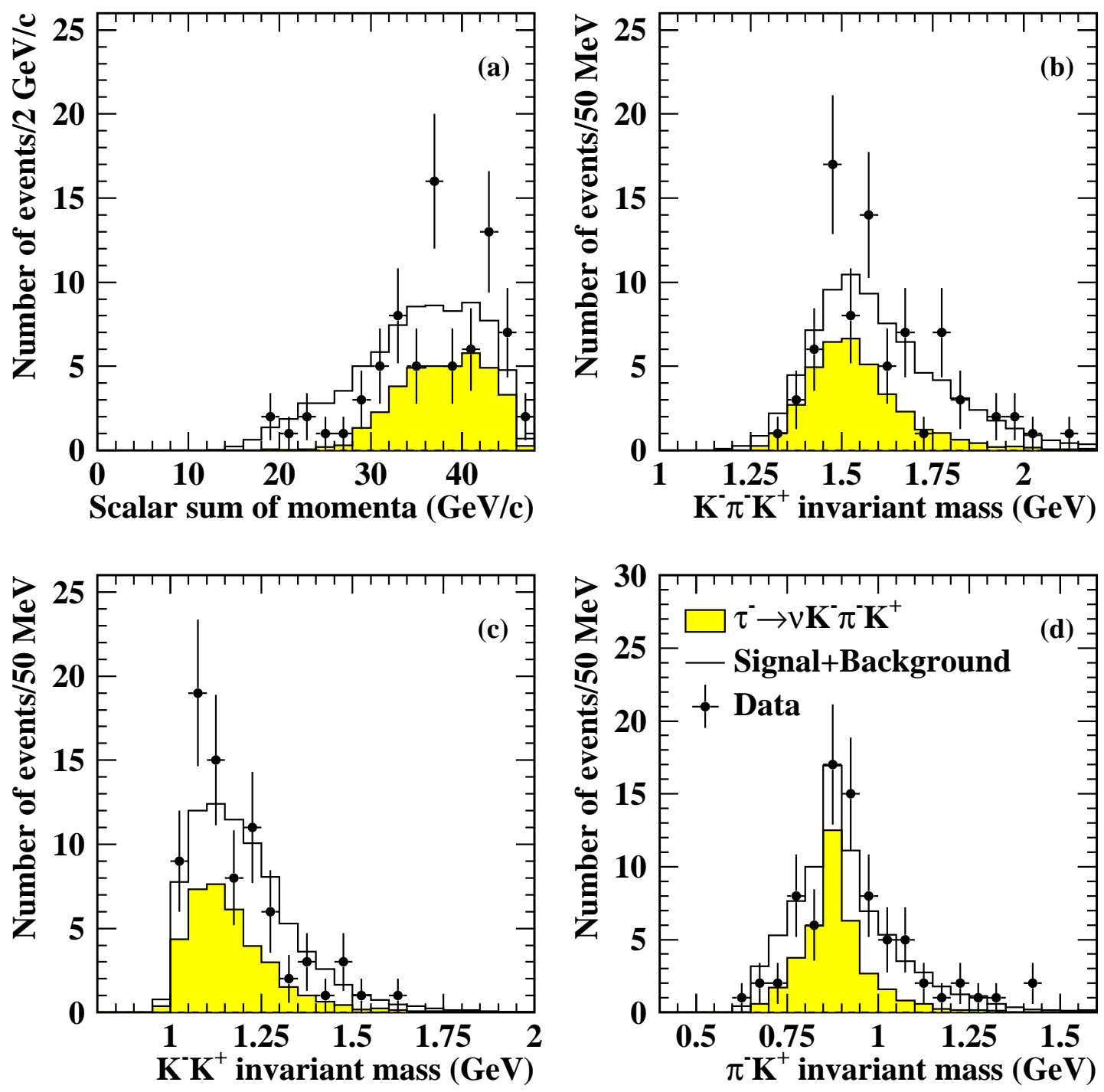

Figure 7: (a) is the $\sum P$ distribution and (b), (c), and (d) are the invariant mass distributions of the data $\tau^{-} \rightarrow \nu_{\tau} \mathrm{K}^{-} \pi^{-} \mathrm{K}^{+}$candidate sample (points). The histogram represents the predicted distribution, in which the normalisation of the background and signal components comes from the results of the binned maximum likelihood fit to the four distributions. The shape of the $\tau^{-} \rightarrow \nu_{\tau} \mathrm{K}^{-} \pi^{-} \mathrm{K}^{+}$signal distribution is simulated in the fit by Monte Carlo events generated by the default version of Tauola 2.4. The shape of the $\tau^{-} \rightarrow \nu_{\tau} \pi^{-} \pi^{-} \pi^{+}\left(\pi^{0}\right)$ background in the distributions is obtained from a data control sample. 